Prepared in cooperation with the Bureau of Land Management

\title{
Assessment of Nonpoint Source Chemical Loading Potential to Watersheds Containing Uranium Waste Dumps and Human Health Hazards Associated with Uranium Exploration and Mining, Red, White, and Fry Canyons, Southeastern Utah, 2007
}

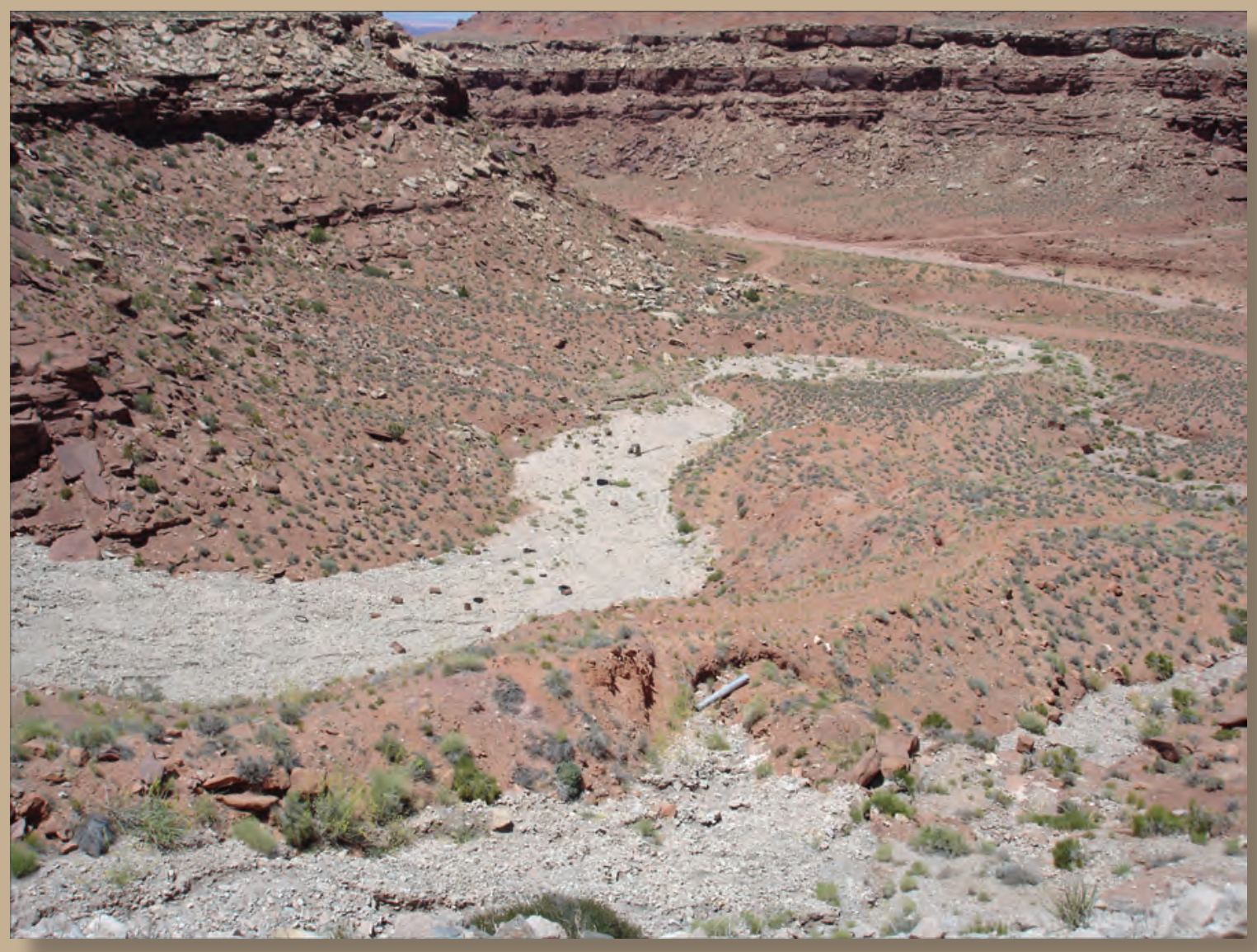

Scientific Investigations Report 2010-5108 
Cover: Path of uranium waste dump material carried away from mine site in Red Canyon, Utah. Photograph taken by Mike Freeman, U.S. Geological Survey, 2007. 


\section{Assessment of Nonpoint Source Chemical Loading Potential to Watersheds Containing Uranium Waste Dumps and Human Health Hazards Associated with Uranium Exploration and Mining, Red, White, and Fry Canyons, Southeastern Utah, 2007}

By Kimberly R. Beisner, Thomas M. Marston, and David L. Naftz, U.S. Geological Survey, Terry Snyder, Bureau of Land Management, and Michael L. Freeman, U.S. Geological Survey

Prepared in cooperation with the Bureau of Land Management

Scientific Investigations Report 2010-5108 


\section{U.S. Department of the Interior \\ KEN SALAZAR, Secretary \\ U.S. Geological Survey \\ Marcia K. McNutt, Director}

\section{U.S. Geological Survey, Reston, Virginia: 2010}

For more information on the USGS — the Federal source for science about the Earth, its natural and living resources, natural hazards, and the environment, visit http://www.usgs.gov or call 1-888-ASK-USGS

For an overview of USGS information products, including maps, imagery, and publications, visit http://www.usgs.gov/ pubprod

To order this and other USGS information products, visit http://store.usgs.gov

Any use of trade, product, or firm names is for descriptive purposes only and does not imply endorsement by the U.S. Government.

Although this report is in the public domain, permission must be secured from the individual copyright owners to reproduce any copyrighted materials contained within this report.

Suggested citation:

Beisner, K.R., Marston, T.M., Naftz, D.L., Snyder, Terry, and Freeman, M.L., 2010, Assessment of nonpoint source chemical loading potential to watersheds containing uranium waste dumps and human health hazards associated with uranium exploration and mining, Red, White, and Fry Canyons, southeastern Utah, 2007: U.S. Geological Survey Scientific Investigations Report 2010-5108, 30 p. 


\section{Contents}

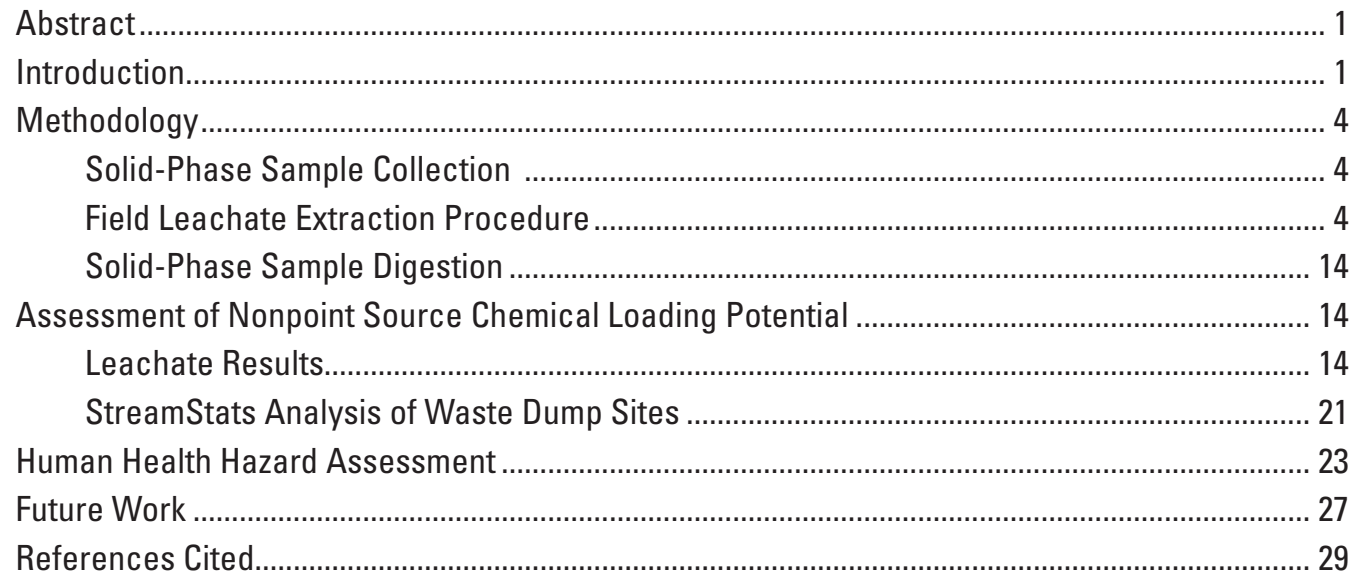

Appendix A. Chemical Analysis of Leachate Samples from Uranium Waste Dump Sites,

Background Sites, and Streambed Sediment Sites, for Major-Ion and Trace-Element

Concentrations, Red, White, and Fry Canyons, southeastern Utah, 2007

\section{Figures}

1. Index map showing areas where uranium waste dumps, streambed sediment, and background sites were sampled, southeastern Utah

2. Map showing location of uranium waste dump, streambed sediment, and background sites in Map 1 where solid-phase material was collected.

3. Map showing location of uranium waste dump, streambed sediment, and background sites in Map 2 where solid-phase material was collected

4. Map showing location of uranium waste dump, streambed sediment, and background sites in Map 3 where solid-phase material was collected

5. Map showing location of uranium waste dump, streambed sediment, and background sites in Map 4 where solid-phase material was collected

6. Map showing location of uranium waste dump, streambed sediment, and background sites in Map 5 where solid-phase material was collected

7. Map showing location of uranium waste dump, streambed sediment, and background sites in Map 6 where solid-phase material was collected

8. Map showing location of uranium waste dump, streambed sediment, and background sites in Map 7 where solid-phase material was collected

9. Map showing location of uranium waste dump, streambed sediment, and background sites in Map 8 where solid-phase material was collected

10. Boxplots showing concentrations of dissolved trace elements in leachate extractions from the 45 uranium waste dump samples collected from Red, White, and Fry Canyons, southeastern Utah, 2007

11. Graph showing concentrations of dissolved arsenic in uranium waste dump leachate samples that exceeded aquatic life and drinking-water-quality standards, Red, White, and Fry Canyons, southeastern Utah, 2007

12. Graph showing concentrations of dissolved beryllium in uranium waste dump leachate samples that exceeded drinking-water-quality standards, Red, White, and Fry Canyons, southeastern Utah, 2007 
13. Graph showing concentrations of dissolved cadmium in uranium waste dump leachate samples that exceeded aquatic life and drinking-water-quality standards, Red, White, and Fry Canyons, southeastern Utah, 2007. 18

14. Graph showing concentrations of dissolved copper in uranium waste dump leachate samples that exceeded aquatic life and drinking-water-quality standards, Red, White, and Fry Canyons, southeastern Utah, 2007

15. Graph showing concentrations of dissolved iron in uranium waste dump leachate samples that exceeded drinking-water-quality standards, Red, White, and Fry Canyons, southeastern Utah, 2007

16. Graph showing concentrations of dissolved manganese in uranium waste dump leachate samples that exceeded drinking-water-quality standards, Red, White, and Fry Canyons, southeastern Utah, 2007

17. Graph showing concentrations of dissolved nickel in uranium waste dump leachate samples that exceeded aquatic life water-quality standards, Red, White, and Fry Canyons, southeastern Utah, 2007

18. Graph showing concentrations of dissolved uranium in uranium waste dump leachate samples that exceeded drinking-water-quality standards, Red, White, and Fry Canyons, southeastern Utah, 2007

19. Graph showing concentrations of dissolved zinc in uranium waste dump leachate samples that exceeded drinking-water-quality standards, Red, White, and Fry Canyons, southeastern Utah, 2007

20. Graph showing relation between leachate and total digestible concentrations for uranium waste dump samples, Red, White, and Fry Canyons, southeastern Utah, 2007

\section{Tables}

1. U.S. Geological Survey site identification and associated Bureau of Land Management tag numbers for abandoned uranium mines with waste dumps, Red, White, and Fry Canyons, southeastern Utah

2. Chemical analysis of process blank samples for selected major-ion and traceelement concentrations, Red, White, and Fry Canyons, southeastern Utah, 2007

3. Chemical analysis of replicate and associated leachate samples from uranium waste dumps for selected major-ion and trace-element concentrations, Red, White, and Fry Canyons, southeastern Utah, 2007.

4. U.S. Environmental Protection Agency drinking-water-quality standards and aquatic life water-quality standards.

5. Drainage area characteristics associated with uranium waste dump samples, Red, White, Blue Notch, and Fry Canyons, southeastern Utah

6. Chemical analysis of total extractible from samples from uranium waste dumps for selected major-ion and trace-element concentrations, Red, White, and Fry Canyons, southeastern Utah, 2007

7. Total extractible uranium concentration calculated from leachate concentration from uranium waste dump samples, Red, White, and Fry Canyons, southeastern Utah, 2007

8. Radium concentrations calculated from total extractible uranium concentrations from uranium waste dump samples, Red, White, and Fry Canyons, southeastern Utah, 2007 


\section{Conversion Factors, Datums, and Abbreviated Water-Quality Units}

\begin{tabular}{|c|c|c|}
\hline Multiply & By & To obtain \\
\hline \multicolumn{3}{|c|}{ Length } \\
\hline centimeter $(\mathrm{cm})$ & 0.3937 & inch (in.) \\
\hline meter $(\mathrm{m})$ & 3.281 & foot $(\mathrm{ft})$ \\
\hline kilometer $(\mathrm{km})$ & 0.6214 & mile (mi) \\
\hline \multicolumn{3}{|c|}{ Area } \\
\hline square kilometer $\left(\mathrm{km}^{2}\right)$ & 0.3861 & square mile $\left(\mathrm{mi}^{2}\right)$ \\
\hline \multicolumn{3}{|c|}{ Volume } \\
\hline liter $(\mathrm{L})$ & 33.82 & ounce, fluid (fl. oz) \\
\hline liter (L) & 0.264 & gallons (gal) \\
\hline \multicolumn{3}{|c|}{ Mass } \\
\hline $\operatorname{gram}(\mathrm{g})$ & 0.03527 & ounce, avoirdupois (oz) \\
\hline \multicolumn{3}{|c|}{ Volumetric Flow Rate } \\
\hline cubic meter per second $\left(\mathrm{m}^{3} / \mathrm{s}\right)$ & 35.314454 & cubic feet per second (cfs) \\
\hline \multicolumn{3}{|c|}{ Concentration } \\
\hline milligram per liter $(\mathrm{mg} / \mathrm{L})$ & 1 & parts per million (ppm) \\
\hline microgram per liter $(\mu \mathrm{g} / \mathrm{L})$ & 1 & parts per billion (ppb) \\
\hline milligram per kilogram $(\mathrm{mg} / \mathrm{kg})$ & 1 & parts per million (ppm) \\
\hline \multicolumn{3}{|c|}{ Radiation Dosage } \\
\hline sievert $(\mathrm{Sv})$ & 100,000 & millirem (mrem) \\
\hline
\end{tabular}

Temperature in degrees Celsius $\left({ }^{\circ} \mathrm{C}\right)$ may be converted to degrees Fahrenheit $\left({ }^{\circ} \mathrm{F}\right)$ as follows:

${ }^{\circ} \mathrm{F}=\left(1.8 \times{ }^{\circ} \mathrm{C}\right)+32$.

Vertical coordinate information is referenced to the North American Vertical Datum of 1988 (NAVD 88).

Horizontal coordinate information is referenced to the North American Datum of 1983 (NAD 83).

Altitude, as used in this report, refers to distance above the vertical datum.

Specific conductance is given in microsiemens per centimeter at 25 degrees Celsius $(\mu \mathrm{S} / \mathrm{cm}$ at $\left.25^{\circ} \mathrm{C}\right)$.

Concentrations of chemical constituents in water are given either in milligrams per liter $(\mathrm{mg} / \mathrm{L})$ or micrograms per liter $(\mu \mathrm{g} / \mathrm{L})$. 


\section{Abbreviated Water-Quality Units}

$\begin{array}{ll}\text { Major constituent } & \text { Abbreviation } \\ \text { Alkalinity } & \mathrm{CaCO}_{3} \\ \text { Calcium } & \mathrm{Ca} \\ \text { Magnesium } & \mathrm{Mg} \\ \text { Potassium } & \mathrm{K} \\ \text { Sodium } & \mathrm{Na} \\ \text { Sulfate } & \mathrm{SO}_{4} \\ & \\ \text { Trace element } & \mathrm{Abbreviation} \\ \text { Aluminum } & \mathrm{Al} \\ \text { Antimony } & \mathrm{Sb} \\ \text { Arsenic } & \mathrm{As} \\ \text { Barium } & \mathrm{Ba} \\ \text { Beryllium } & \mathrm{Be} \\ \text { Cadmium } & \mathrm{Cd} \\ \text { Chromium } & \mathrm{Cr} \\ \text { Cobalt } & \mathrm{Co} \\ \text { Copper } & \mathrm{Cu} \\ \text { Iron } & \mathrm{Fe} \\ \text { Lead } & \mathrm{Pb} \\ \text { Manganese } & \mathrm{Mn} \\ \text { Molybdenum } & \mathrm{Mo} \\ \text { Nickel } & \mathrm{Ni} \\ \text { Selenium } & \mathrm{Se} \\ \text { Silver } & \mathrm{Ag} \\ \text { Thallium } & \mathrm{Tl} \\ \text { Uranium } & \mathrm{U} \\ \text { Vanadium } & \mathrm{V} \\ \text { Zinc } & \mathrm{Zn} \\ & \end{array}$




\title{
Assessment of Nonpoint Source Chemical Loading Potential to Watersheds Containing Uranium Waste Dumps and Human Health Hazards Associated with Uranium Exploration and Mining, Red, White, and Fry Canyons, Southeastern Utah, 2007
}

\author{
By Kimberly R. Beisner, Thomas M. Marston, and David L. Naftz, U.S. Geological Survey, Terry Snyder, \\ Bureau of Land Management, and Michael L. Freeman, U.S. Geological Survey
}

\begin{abstract}
During May, June, and July 2007, 58 solid-phase samples were collected from abandoned uranium mine waste dumps, background sites, and adjacent streambeds in Red, White, and Fry Canyons in southeastern Utah. The objectives of this sampling program were to (1) assess the nonpoint-source chemical loading potential to ephemeral and perennial drainage basins from uranium waste dumps and (2) assess potential effects on human health due to recreational activities on and around uranium waste dumps on Bureau of Land Management property. Uranium waste-dump samples were collected using solid-phase sampling protocols. After collection, solid-phase samples were homogenized and extracted in the laboratory using a leaching procedure. Filtered ( 0.45 micron) water samples were obtained from the field leaching procedure and were analyzed for major and trace elements at the Inductively Coupled Plasma-Mass Spectrometry Metals Analysis Laboratory at the University of Utah. A subset of the solid-phase samples also were digested with strong acids and analyzed for major ions and trace elements at the U.S. Geological Survey Geologic Division Laboratory in Denver, Colorado.

For the initial ranking of chemical loading potential for uranium waste dumps, results of leachate analyses were compared with existing aquatic-life and drinking-waterquality standards. To assess potential effects on human health, solid-phase digestion values for uranium were compared to soil screening levels (SSL) computed using the computer model RESRAD 6.5 for a probable concentration of radium. One or more chemical constituents exceeded aquatic life and drinking-water-quality standards in approximately 64 percent (29/45) of the leachate samples extracted from uranium waste dumps. Most of the uranium waste dump sites with elevated
\end{abstract}

trace-element concentrations in leachates were located in Red Canyon. Approximately 69 percent (31/45) of the strong acid digestible soil concentration values were greater than a calculated SSL. Uranium waste dump sites with elevated leachate and total digestible concentrations may need to be further investigated to determine the most appropriate remediation method.

\section{Introduction}

Red, White, and Fry Canyons in San Juan County, Utah, in the Colorado Plateau physiographic province encompass approximately $400 \mathrm{mi}^{2}$ of primarily Bureau of Land Management (BLM) property (fig. 1). Red, White, and Fry Canyons generally are linear with a drainage direction toward the northwest and altitudes ranging from 3,700 to 7,500 $\mathrm{ft}$. Exposed rocks range in age from the Permian (Cutler Formation) through the Jurassic (Navajo Sandstone). Most of the land is covered by ephemeral streams that only contribute runoff during rainfall and snowmelt events. Red Canyon and a smaller canyon, Blue Notch Canyon, drain into Good Hope Bay on Lake Powell. Fry Canyon drains into White Canyon, which enters Lake Powell north of Good Hope Bay.

Red, White, and Fry Canyons are characterized by mild winters and hot summers. Most of the precipitation falls as rain from convective thunderstorms that typically occur between August and October. This arid region receives approximately 6 to 8 in. of precipitation at the lower altitudes and approximately 8 to $10 \mathrm{in}$. of precipitation at the higher altitudes (Natural Resources Conservation Service, 1998). 


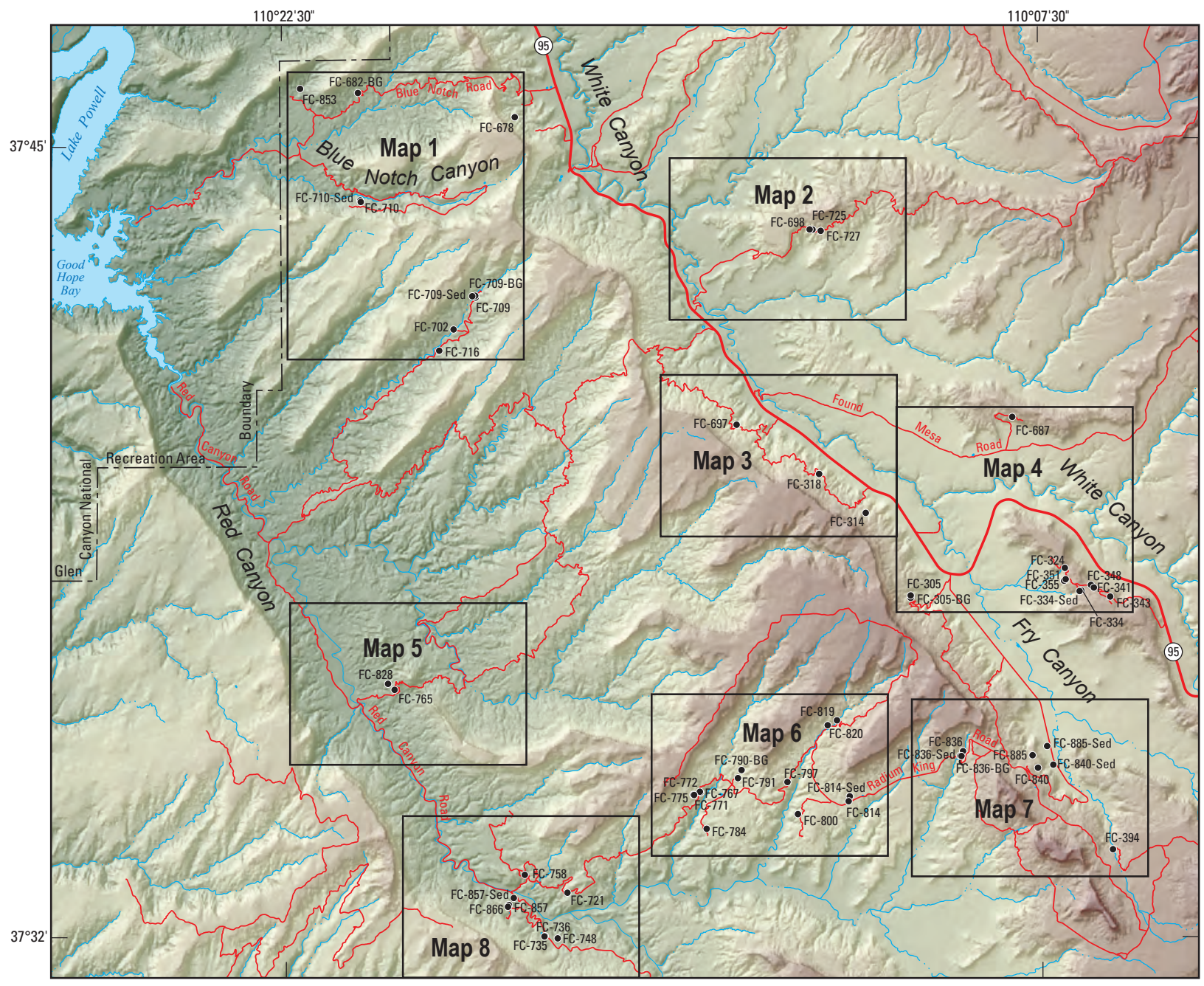

Base from U.S. Geological Survey digital data

Hillshade from 30-meter USGS National Elevation Dataset, 1999

Universal Transverse Mercator projection, Zone 12
North American Datum of 1983

0

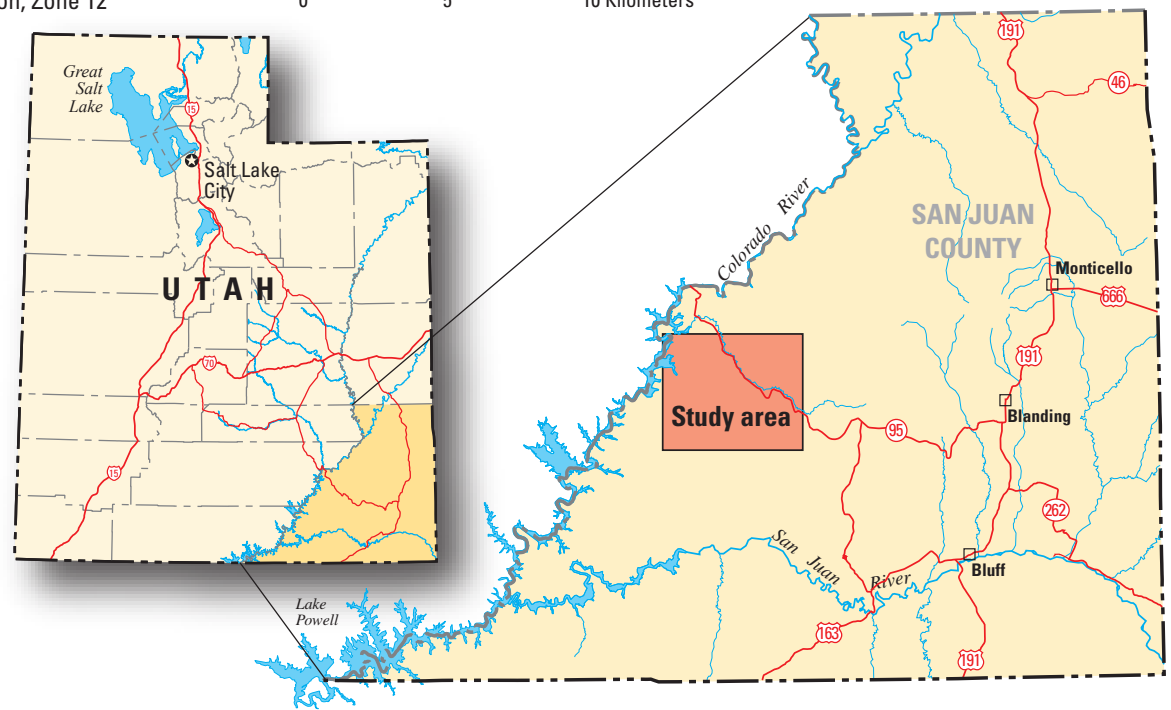

Figure 1. Areas where uranium waste dumps, streambed sediment, and background sites were sampled, southeastern Utah. 
Several areas in southern Utah experienced an increase in uranium mining and exploration in the 1950s. Most of the mines extracted uranium ore from the Triassic Chinle Formation, which is overlain by the Wingate Sandstone and is underlain by the Moenkopi Formation. At the time of publication, thousands of abandoned uranium mine waste dumps exist throughout the State of Utah, with many of the properties located on lands managed by the BLM.

Approximately 65 uranium waste dump sites and adits have been inventoried by the BLM throughout Red, White, and Fry Canyons (Terry Snyder, Bureau of Land Management, written commun., 2007); however, several of those mines were not accessible during this study so only 45 abandoned uranium mines with waste dumps were sampled for this study (table 1). These abandoned uranium mine waste dumps have unique characteristics that make it difficult to quantify nonpoint source pollution contributions to specific watersheds. These characteristics include (1) locations that are primarily in watersheds with ephemeral streams, (2) radioactive sands and fine particulates that are radioactive for hundreds of thousands of years, (3) intense rainfall and snowmelt events that can mobilize and transport mine waste with associated radioactive material and trace elements long distances during relatively short periods, and (4) remote locations that do not allow for cost effective water and suspended-sediment sampling during storm and snowmelt runoff events.

Sample collection from uranium waste dump sites during May, June, and July 2007 was completed by the U.S. Geological Survey (USGS) in cooperation with the BLM. The objectives of this sampling program were to (1) assess the nonpoint source chemical loading potential to ephemeral and perennial watersheds from uranium waste dumps and (2) assess potential impacts on human health due to recreational activities on and around uranium waste dumps on Bureau of Land Management property. Initial ranking of the chemical loading potential of each sample was done by comparing the leachate composition with existing U.S. Environmental Protection Agency (USEPA) water-quality standards for drinking water and aquatic life. Solid-phase acid extractable results were compared to soil screening levels using USEPA factors for carcinogenic effects to human health.

Table 1. U.S. Geological Survey site identification and associated Bureau of Land Management tag numbers for abandoned uranium mines with waste dumps, Red, White, and Fry Canyons, southeastern Utah.

[USGS Site ID, U.S. Geological Survey Site Identification; BLM, Bureau of Land Management; FC, Fry Canyon area]

\begin{tabular}{|c|c|c|c|}
\hline USGS Site ID & BLM Tag Number & USGS Site ID & BLM Tag Number \\
\hline FC-305 & UT67800110 and UT67800123 & FC-736 & UT67800085 \\
\hline FC-314 & UT67800126 & FC-748 & UT67800087 \\
\hline FC-318 & UT67800127 & FC-758 & UT67800088 \\
\hline FC-324 & UT67800117 & FC-765 & UT67800090 \\
\hline FC-334 & UT67800119 & FC-767 & UT67800091 \\
\hline FC-341 & UT67800119 & FC-771 & UT67800091 \\
\hline FC-343 & UT67800124 & FC-772 & UT67800091 \\
\hline FC-348 & UT67800119 & FC-775 & UT67800091 \\
\hline FC-351 & UT67800116 & FC-784 & UT67800092 \\
\hline FC-355 & UT67800118 & FC-791 & UT67800094 \\
\hline FC-394 & UT67800125 & FC-797 & UT67800095 \\
\hline FC-678 & UT67800065 & FC- 800 & UT67800096 \\
\hline FC-687 & UT67800069 & FC-814 & UT67800099 \\
\hline FC-697 & UT67800071 & FC-819 & UT67800100 \\
\hline FC-698 & UT67800072 & FC-820 & UT67800101 \\
\hline FC-702 & UT67800076 & FC-828 & UT67800102 \\
\hline FC-709 & UT67800076 & FC-836 & UT67800106 \\
\hline FC-710 & UT67800077 & FC-840 & UT67800107 \\
\hline FC-716 & UT67800078 & FC-853 & UT67800114 \\
\hline FC-721 & UT67800079 & FC-857 & UT67800121 \\
\hline FC-725 & UT67800082 & FC-866 & UT67800121 \\
\hline FC-727 & UT67800083 & FC-885 & UT67800108 \\
\hline
\end{tabular}




\section{Methodology}

\section{Solid-Phase Sample Collection}

Fifty-eight samples from 45 uranium waste dump sites, 8 streambed-sediment sites, and 5 background sites were collected in May, June, and July 2007, using a modified version of the solid-phase sampling methods outlined by Smith and others (2000) and Hageman and Briggs (2000). The general locations of uranium waste dump, background, and streambed-sediment sites that were sampled are shown in figure 1, and more detailed maps of the sampled sites are shown in figures 2-9. Because of the steep embankments of the uranium waste dumps, sampling generally took place from the safest and most accessible location. A few of the BLM inventoried sites that were unsafe to access were not sampled. Background samples were collected in the same geologic layer adjacent to sampled uranium waste dump sites. Approximately one background sample was collected for every 10 uranium waste dump samples to collect a representative selection of background samples. Streambed sediment samples were collected from ephemeral stream channels downstream from sampled uranium waste dump sites.

Samples from uranium waste dump and geologic background sites consisted of 30 scoops of soil collected from as deep as $15 \mathrm{~cm}$ using a plastic trowel. Scoops of soil were combined into 5-gallon plastic buckets, sealed with lids, and labeled. Streambed-sediment samples were collected along ephemeral stream channels using a plastic trowel, and these samples consisted of 10 scoops from as deep as $15 \mathrm{~cm}$. Each streambed scoop location was chosen at random from a 10- to 30-m transect across the channel downstream of a sampled uranium waste dump site. Scoops of sediment were compiled into 1-gallon plastic Ziploc $\AA$ bags and labeled.

Uranium waste dump sites were labeled FC (Fry Canyon) and background sites were given the same number as the associated uranium waste dump and were labeled FC-BG (Fry Canyon-Background). Streambed-sediment samples were labeled FC-SED (Fry Canyon-Sediment). Fry Canyon sample IDs that were assigned to the abandoned uranium mines by the USGS have been paired with the site IDs assigned by the BLM and are shown in table 1.

All sampling equipment was thoroughly cleaned in the field between each sampling location. An all-plastic multi-purpose sprayer was used to wash plastic trowels, collection buckets, and lids. Each item was rinsed three times with de-ionized water, wiped with a clean towel, and allowed to air dry before use at the next site.

\section{Field Leachate Extraction Procedure}

In September 2007, after the collection of solid-phase samples, aqueous phase leachate samples were extracted in the laboratory using a field-leaching procedure. Solid-phase samples were thoroughly mixed using a plastic trowel, after which approximately $800 \mathrm{~g}$ of sediment were measured using a pan balance, transferred to a 1-L glass beaker, and allowed to air dry. The dried sample was then sieved through a 2-mm stainless-steel mesh screen.

Fifty grams of sieved sample were added to $1,000 \mathrm{~g}$ of deionized water to create a 1:20 solid-to-liquid ratio. The sieved sample and water were then mixed in a precleaned 1.5-L wide-mouth high-density polyethylene (HDPE) Nalgene ${ }^{\circledR}$ bottle and agitated for 5 minutes on a shaker table operating at 170 oscillations per minute. After shaking, the samples were allowed to settle for 1 hour prior to filtration.

The leachate water was filtered through a 0.45 -micron high-capacity Versapor ${ }^{\circledR}$ membrane capsule filter (GeoTech dispos-a-filter ${ }^{\mathrm{TM}}$ ) using precleaned tubing and a peristaltic pump. For each leachate sample, the filtered water samples were collected in one 125-mL HDPE bottle and one 125-mL acid-rinsed HDPE bottle. The samples collected in the $125-\mathrm{mL}$ HDPE bottles were sent to the USGS National Water Quality Laboratory (NWQL) in Denver, Colorado, for $\mathrm{SO}_{4}$ and alkalinity analysis. The samples collected in 125-mL acidrinsed HDPE bottles were preserved with 1-mL Ultrex-grade 7.7N nitric acid and sent to the Inductively Coupled PlasmaMass Spectrometry (ICP-MS) Metals Analysis Laboratory at the University of Utah for major-ion and trace-element analysis (Ag, Al, As, Ba, Be, Ca, Cd, Co, Cr, Cu, Fe, K, Mg, $\mathrm{Mn}, \mathrm{Mo}, \mathrm{Na}, \mathrm{Ni}, \mathrm{Pb}, \mathrm{Sb}, \mathrm{Se}, \mathrm{Tl}, \mathrm{U}, \mathrm{V}$, and $\mathrm{Zn}$ ). The remaining unfiltered aliquot of water was then decanted and used to measure specific conductance and $\mathrm{pH}$.

Bottles and tubing used for sample extraction were cleaned according to the USGS inorganic constituents cleaning procedures outlined in Wilde (2004). One process blank and one replicate sample were processed after every 10 leachate samples. All process blank samples were labeled as FC BLANK and given an individual extraction time and date. Blanks and replicates were analyzed at the ICP-MS Metals Analysis Laboratory at the University of Utah and the USGS NWQL. Process blank samples followed the same extraction procedures as leachate samples in the laboratory, but process blank samples were agitated without any sediment in the $1.5-\mathrm{L}$ Nalgene ${ }^{\circledR}$ bottles.

Results of chemical analysis for the five process blank samples are presented in table 2. Most of the trace-element concentrations for the process blank samples were less than the lower reporting limits; however, concentrations for $\mathrm{Ag}, \mathrm{Cd}$, $\mathrm{Co}, \mathrm{Cr}, \mathrm{Cu}, \mathrm{Fe}, \mathrm{Mn}, \mathrm{Mo}$, and $\mathrm{Sb}$ exceeded the lower reporting limits. All Ag concentrations were equal to or less than the lower reporting limit with the exception of one blank sample $(0.008 \mu \mathrm{g} / \mathrm{L})$. All Cd concentrations were equal to or less than the lower reporting limit with the exception of one blank sample $(0.04 \mu \mathrm{g} / \mathrm{L})$. Concentrations of Co ranged from 0.11 to $0.26 \mu \mathrm{g} / \mathrm{L}$ with a median concentration of $0.18 \mu \mathrm{g} / \mathrm{L}$. All Cr concentrations were less than the lower reporting limit with the exception of one blank sample $(0.06 \mu \mathrm{g} / \mathrm{L})$. All Cu concentrations were less than the lower reporting limit with the exception of one blank sample $(0.98 \mu \mathrm{g} / \mathrm{L})$. All Fe concentrations were less than the lower reporting limit with the 


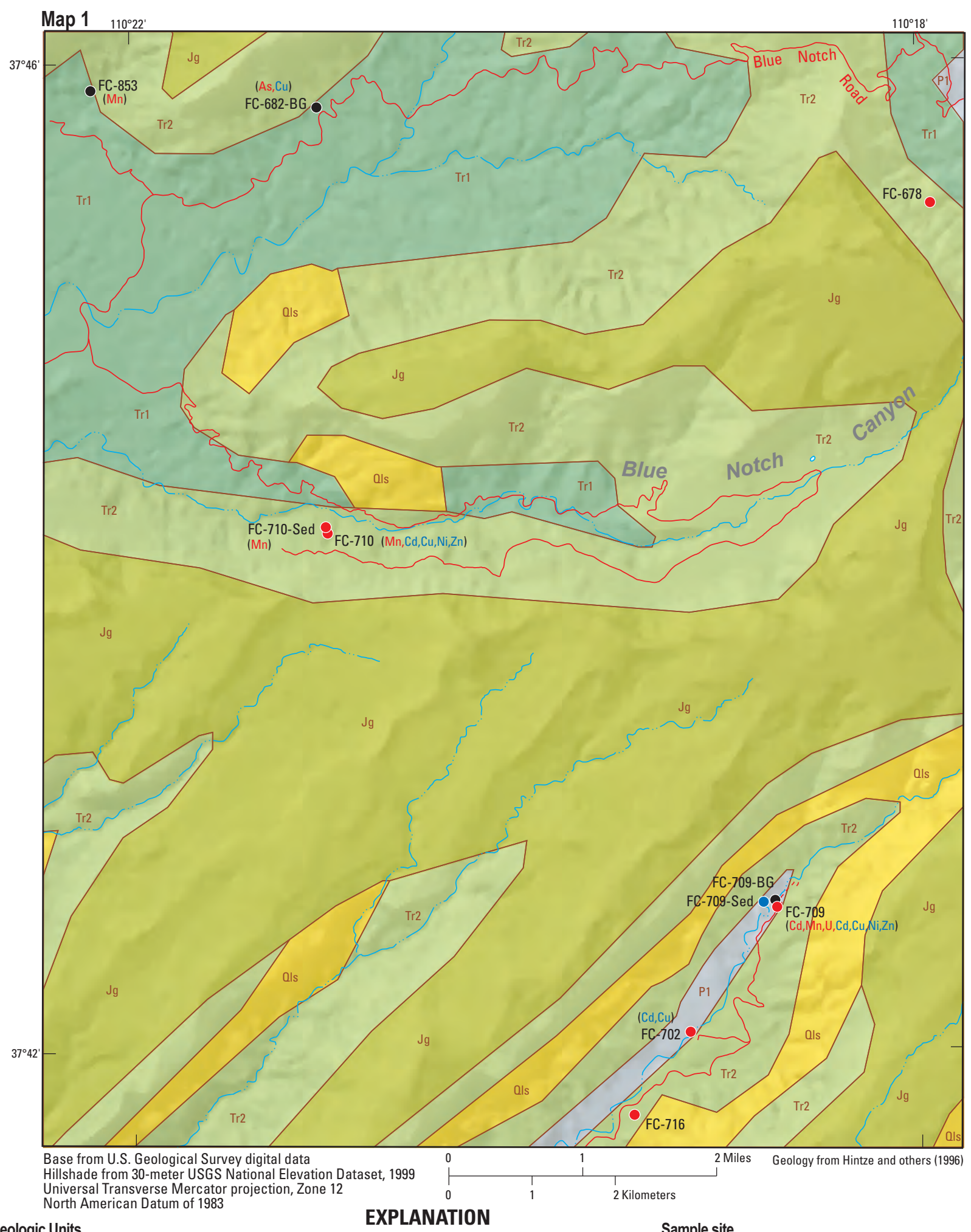

\section{Geologic Units}

Qls Quaternary, Landslide deposits - surficial deposits

$\mathrm{Jg}$ Jurassic, Glen Canyon Group-Navajo Sandstone, Kayenta Formation, Wingate Sandstone

Tr2 Triassic, Chinle Formation-Shinarump Member

Tr1 Triassic, Moenkopi Formation

P1 Permian—White Rim Sandstone, DeChelly Sandstone, Organ Rock Shale, Cedar Mesa Sandstone, Halgaito Formation, Elephant Canyon Formation

\section{Sample site}

FC-716 Site ID, refer to table 4 for site information (Mn,Cu,Ni) Constituents listing for those that exceed water-quality standards, see table 5. Mn indicates drinking-water standard, Cu indicates aquatic life standard

- Geologic background site

- Streambed sediment site

- Uranium waste dump site

Figure 2. Location of uranium waste dump, streambed sediment, and background sites in Map 1 where solid-phase material was collected. 


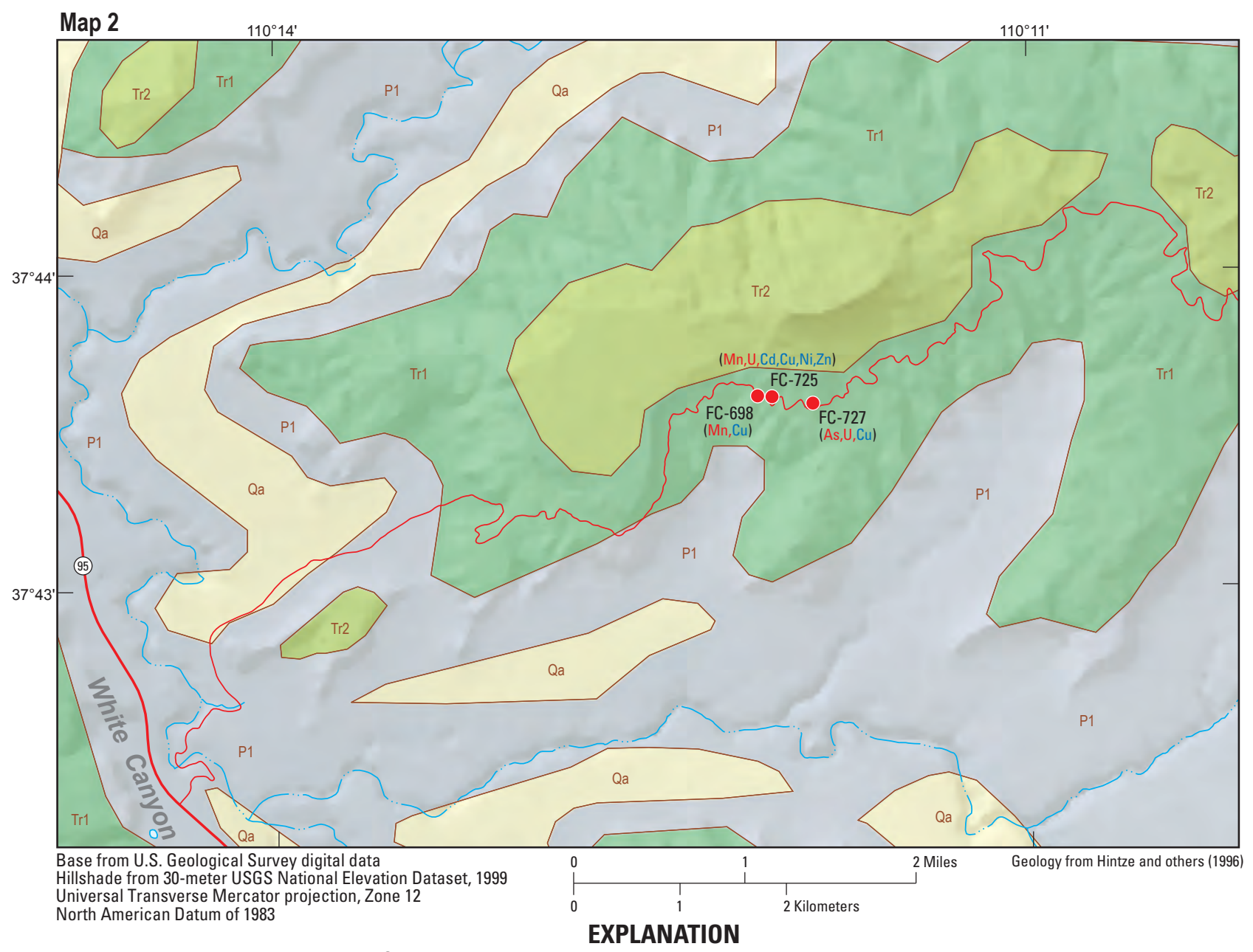

Geologic Units

Qa Quaternary, Surficial deposits-Alluvium and colluvium deposits

$T \mathrm{~T} 2$ Triassic, Chinle Formation-Shinarump Member

Tr1 Triassic, Moenkopi Formation

P1 Permian-White Rim Sandstone, DeChelly Sandstone, Organ Rock Shale, Cedar Mesa Sandstone, Halgaito Formation, Elephant Canyon Formation

Sample site

FC-698 Site ID, refer to table 4 for site information

$(\mathrm{Mn}, \mathrm{Cu}, \mathrm{Ni})$ Constituents listing for those that exceed water-quality standards, see table 5.

$\mathrm{Mn}$ indicates drinking-water standard, $\mathrm{Cu}$ indicates aquatic life standard

- Geologic background site

- Streambed sediment site

- Uranium waste dump site

Figure 3. Location of uranium waste dump, streambed sediment, and background sites in Map 2 where solid-phase material was collected. 


\section{Map 3}

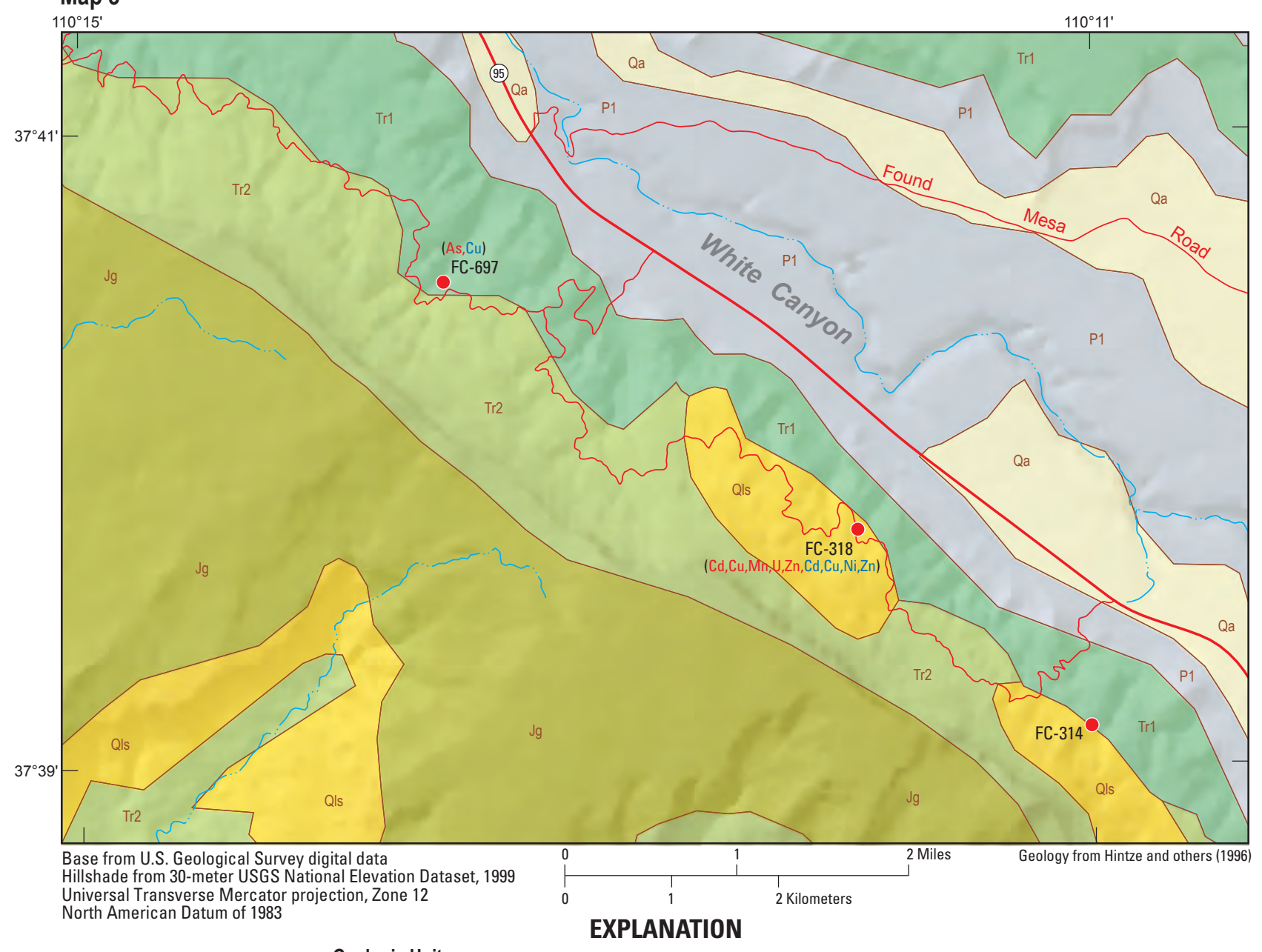

\section{Geologic Units}

\section{EXPLANATION}

Quaternary, Surficial deposits-Alluvium and colluvium deposits

Quaternary, Landslide deposits-Surficial deposits

Jurassic, Glen Canyon Group-Navajo Sandstone, Kayenta Formation, Wingate Sandstone

Triassic, Chinle Formation-Shinarump Member

$T$ Tr1 Triassic, Moenkopi Formation

P1 Permian-White Rim Sandstone, DeChelly Sandstone, Organ Rock Shale, Cedar Mesa Sandstone, Halgaito Formation, Elephant Canyon Formation

\section{Sample site}

FC-318 Site ID, refer to table 4 for site information

$(\mathrm{Mn}, \mathrm{Cu}, \mathrm{Ni})$ Constituents listing for those that exceed water-quality standards, see table 5. Mn indicates drinking-water standard, $\mathrm{Cu}$ indicates aquatic life standard

- Geologic background site

- Streambed sediment site

- Uranium waste dump site

Figure 4. Location of uranium waste dump, streambed sediment, and background sites in Map 3 where solid-phase material was collected. 


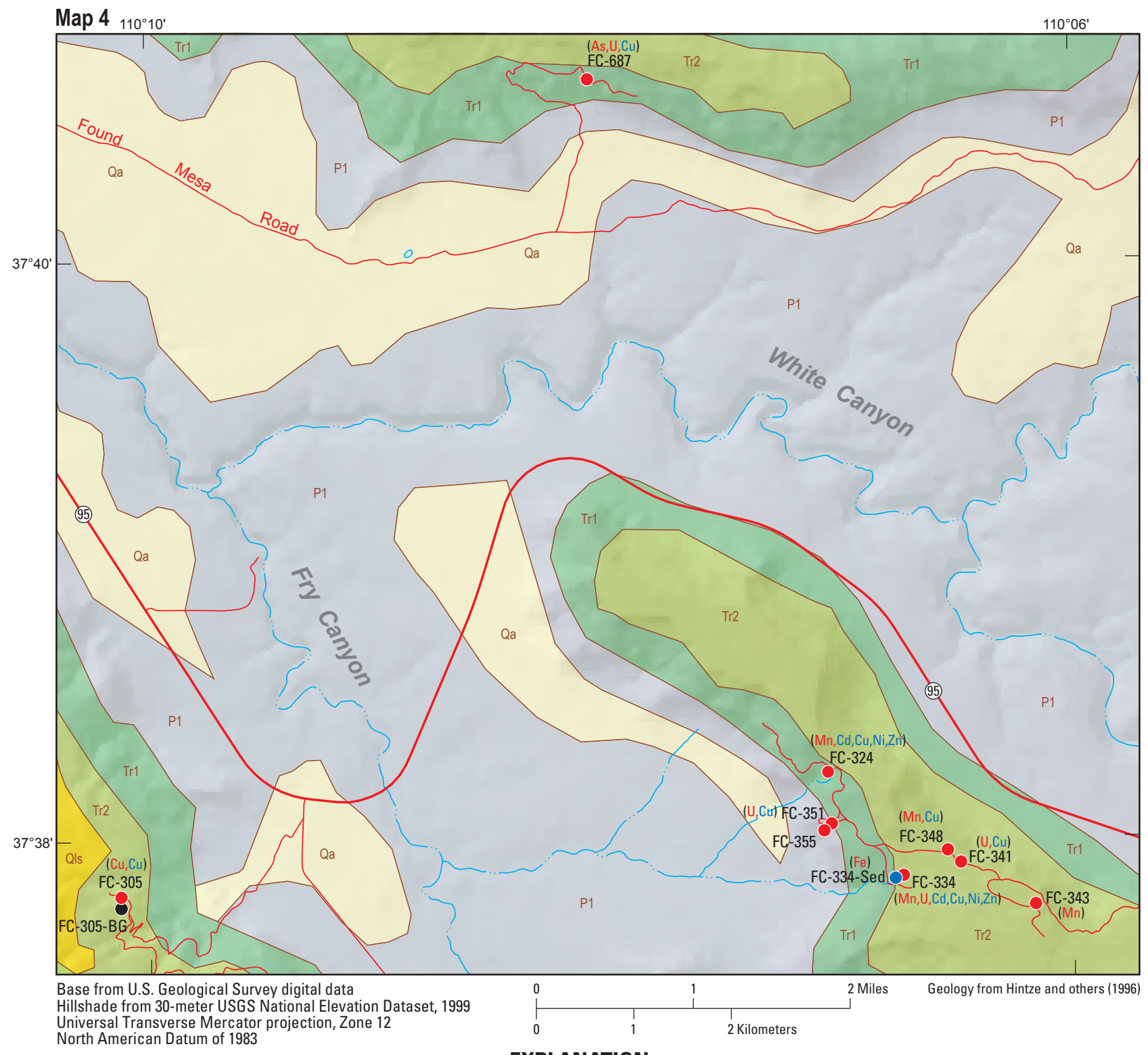

North American Datum of 1983

Geologic Units

EXPLANATION

Quaternary, surficial deposits-Alluvium and colluvium deposits

Quaternary, surficial deposits-Landslide deposits

Triassic, Chinle Formation-Shinarump Member

Triassic, Moenkopi Formation

Permian-White Rim Sandstone, DeChelly Sandstone, Organ Rock Shale, Cedar Mesa Sandstone, Halgaito Formation, Elephant Canyon Formation

\section{Sample site}

FC-305 Site ID, refer to table 4 for site information

$(\mathrm{Mn}, \mathrm{Cu}, \mathrm{Ni})$ Constituents listing for those that exceed water-quality standards, see table 5. Mn indicates drinking-water standard, Cu indicates aquatic life standard

- Geologic background site

- Streambed sediment site

- Uranium waste dump site

Figure 5. Location of uranium waste dump, streambed sediment, and background sites in Map 4 where solid-phase material was collected. 


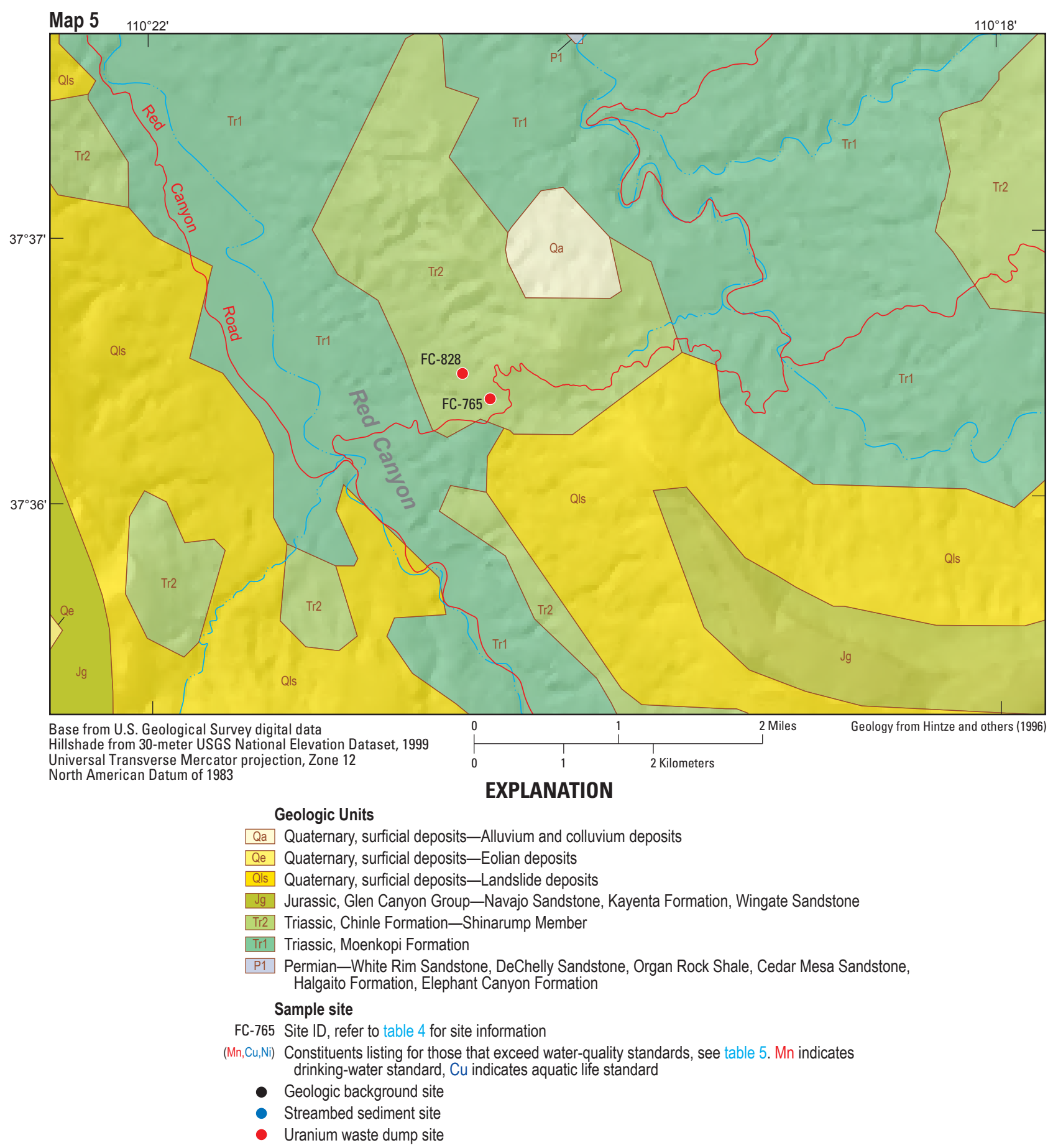

Figure 6. Location of uranium waste dump, streambed sediment, and background sites in Map 5 where solid-phase material was collected. 


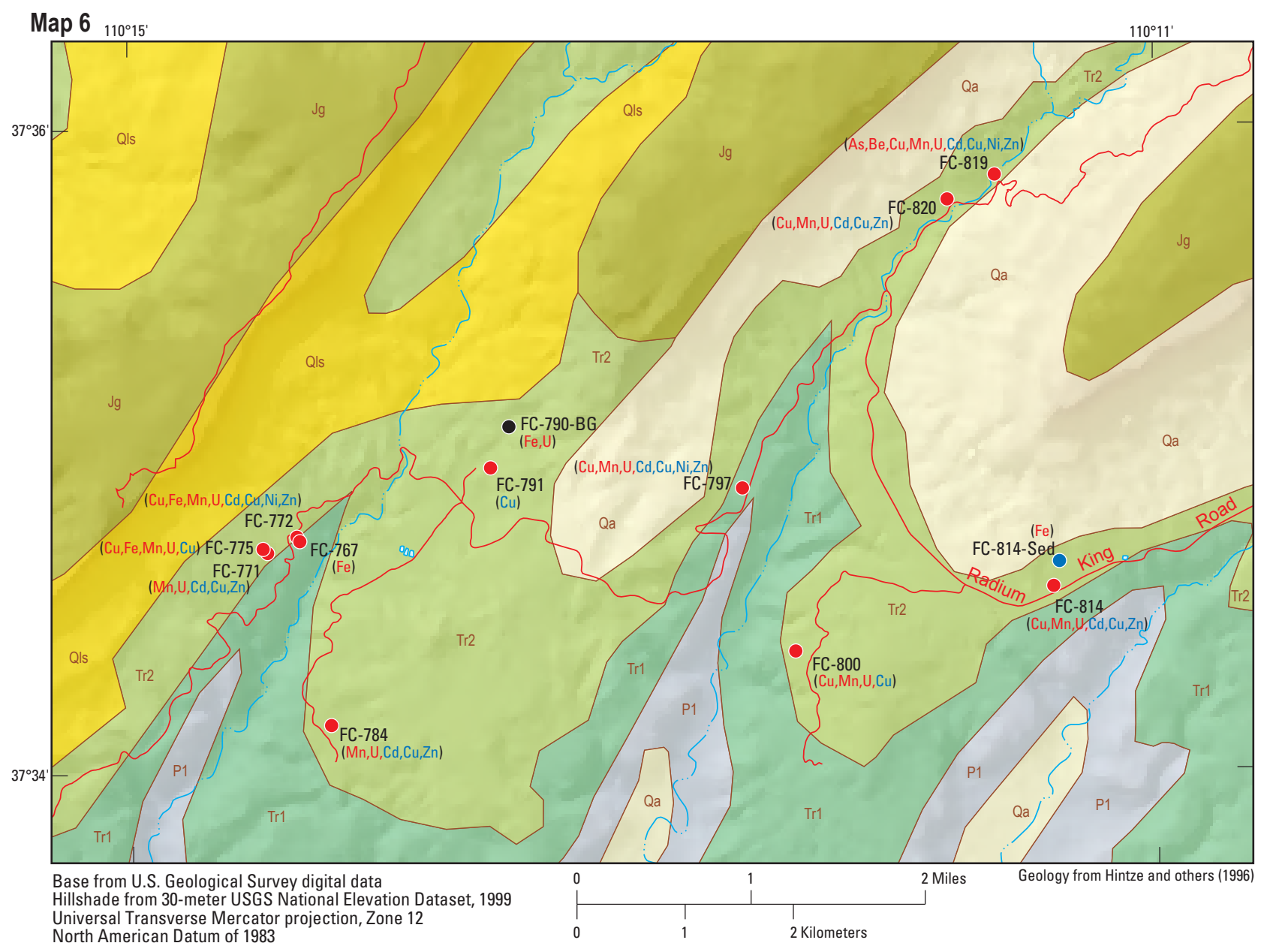

Universal Transverse Mercator projection, Zone 12

\section{EXPLANATION}

Geologic Units

Qa Quaternary, surficial deposits-Alluvium and colluvium deposits

Qls Quaternary, surficial deposits—Landslide deposits

$\mathrm{Jg}$ Jurassic, Glen Canyon Group-Navajo Sandstone, Kayenta Formation, Wingate Sandstone

Tr2 Triassic, Chinle Formation-Shinarump Member

Tr1 Triassic, Moenkopi Formation

P1 Permian -White Rim Sandstone, DeChelly Sandstone, Organ Rock Shale, Cedar Mesa Sandstone, Halgaito Formation, Elephant Canyon Formation

Sample site

FC-765 Site ID, refer to table 4 for site information

(Mn, $\mathrm{Cu}, \mathrm{Ni})$ Constituents listing for those that exceed water-quality standards, see table 5. Mn indicates drinking-water standard, Cu indicates aquatic life standard

- Geologic background site

- Streambed sediment site

- Uranium waste dump site

Figure 7. Location of uranium waste dump, streambed sediment, and background sites in Map 6 where solid-phase material was collected. 


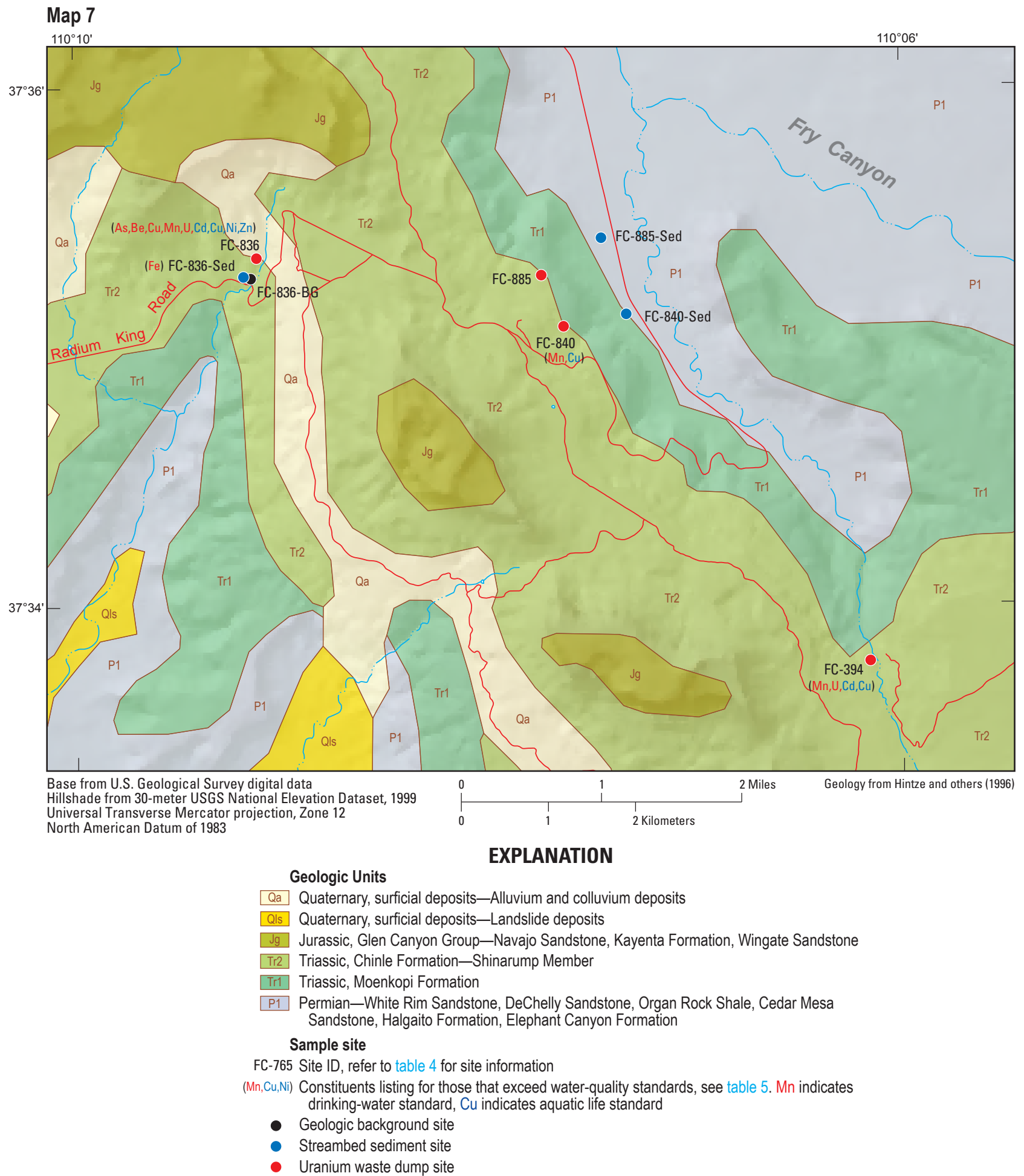

Figure 8. Location of uranium waste dump, streambed sediment, and background sites in Map 7 where solid-phase material was collected. 


\section{Map 8}

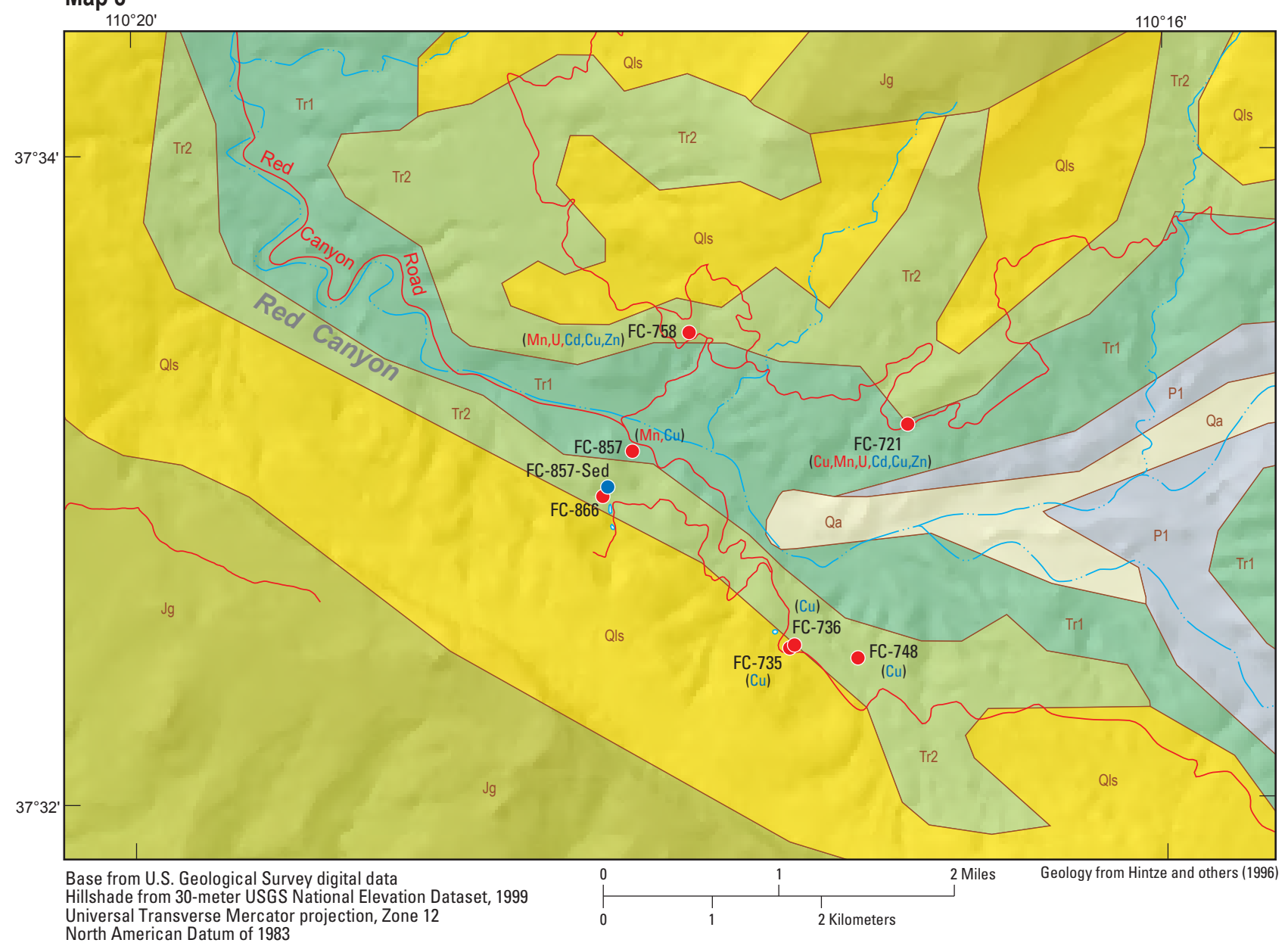

Hillshade from 30 -meter USGS National Elevation Dataset, 1999
Universal Transverse Mercator projection, Zone 12

North American Datum of 1983

\section{EXPLANATION}

Geologic Units

Quaternary, surficial deposits-Alluvium and colluvium deposits

S Quaternary, surficial deposits - Landslide deposits

Jurassic, Glen Canyon Group—Navajo Sandstone, Kayenta Formation, Wingate Sandstone

2 Triassic, Chinle Formation-Shinarump Member

Triassic, Moenkopi Formation

1 Permian-White Rim Sandstone, DeChelly Sandstone, Organ Rock Shale, Cedar Mesa Sandstone, Halgaito Formation, Elephant Canyon Formation

Sample site

FC-765 Site ID, refer to table 4 for site information

$(\mathrm{Mn}, \mathrm{Cu}, \mathrm{Ni})$ Constituents listing for those that exceed water-quality standards, see table 5. Mn indicates drinking-water standard, Cu indicates aquatic life standard

- Geologic background site

- Streambed sediment site

- Uranium waste dump site

Figure 9. Location of uranium waste dump, streambed sediment, and background sites in Map 8 where solid-phase material was collected. 
Table 2. Chemical analysis of process blank samples for selected major-ion and trace-element concentrations, Red, White, and Fry Canyons, southeastern Utah, 2007.

[Samples were analyzed at the Inductively Coupled Plasma-Mass Spectrometry Metals Analysis Laboratory at the University of Utah. Site ID, site identification; MDT, Mountain Daylight Time; fil, filtered sample; lab, laboratory analysis; mg/L, milligrams per liter; $\mu \mathrm{g} / \mathrm{L}$, micrograms per liter; FC, Fry Canyon area; <, less than lower reporting limit]

\begin{tabular}{|c|c|c|c|c|c|c|c|c|c|}
\hline Site ID & $\begin{array}{c}\text { Sample } \\
\text { extraction } \\
\text { date }\end{array}$ & $\begin{array}{c}\text { Time } \\
\text { (MDT) }\end{array}$ & $\begin{array}{l}\text { Alkalinity, fil, } \\
\text { lab (mg/L as } \\
\left.\mathrm{CaCO}_{3}\right)\end{array}$ & $\begin{array}{l}\text { Calcium, } \\
\text { dissolved } \\
\text { (mg/L) }\end{array}$ & $\begin{array}{l}\text { Magnesium, } \\
\text { dissolved } \\
\text { (mg/L) }\end{array}$ & $\begin{array}{l}\text { Potassium, } \\
\text { dissolved } \\
\text { (mg/L) }\end{array}$ & $\begin{array}{c}\text { Sodium, } \\
\text { dissolved } \\
\text { (mg/L) }\end{array}$ & $\begin{array}{c}\text { Sulfate, } \\
\text { dissolved } \\
\text { (mg/L as } \\
\mathrm{SO}_{4} \text { ) }\end{array}$ & $\begin{array}{c}\text { Aluminum, } \\
\text { dissolved } \\
(\mu \mathrm{g} / \mathrm{L})\end{array}$ \\
\hline FC-BLANK & $9 / 17 / 2007$ & 1405 & $<5.0$ & ${ }^{1} 0.04$ & $<0.004$ & $<0.02$ & $<0.06$ & $<0.18$ & $<1.0$ \\
\hline FC-BLANK & $9 / 19 / 2007$ & 1425 & $<5.0$ & $<.02$ & .003 & $<.02$ & $<.06$ & $<.18$ & $<1.0$ \\
\hline FC-BLANK & $9 / 20 / 2007$ & 1205 & $<5.0$ & .01 & $<.004$ & $<.02$ & $<.06$ & $<.18$ & $<1.0$ \\
\hline FC-BLANK & $9 / 20 / 2007$ & 1305 & $<5.0$ & $<.01$ & $<.004$ & $<.02$ & $<.06$ & $<.18$ & $<1.0$ \\
\hline FC-BLANK & $9 / 21 / 2007$ & 1130 & $<5.0$ & $<.02$ & $<.004$ & $<.02$ & $<.06$ & $<.18$ & $<1.0$ \\
\hline
\end{tabular}

\begin{tabular}{|c|c|c|c|c|c|c|c|c|c|}
\hline Site ID & $\begin{array}{c}\text { Sample } \\
\text { extraction } \\
\text { date }\end{array}$ & $\begin{array}{c}\text { Time } \\
\text { (MDT) }\end{array}$ & $\begin{array}{l}\text { Antimony, } \\
\text { dissolved } \\
(\mu \mathrm{g} / \mathrm{L}\end{array}$ & $\begin{array}{c}\text { Arsenic, } \\
\text { dissolved } \\
(\mu \mathrm{g} / \mathrm{L})\end{array}$ & $\begin{array}{c}\text { Barium, } \\
\text { dissolved } \\
(\mu \mathrm{g} / \mathrm{L})\end{array}$ & $\begin{array}{l}\text { Beryllium, } \\
\text { dissolved } \\
(\mu \mathrm{g} / \mathrm{L})\end{array}$ & $\begin{array}{c}\text { Cadmium, } \\
\text { dissolved } \\
(\mu \mathrm{g} / \mathrm{L})\end{array}$ & $\begin{array}{c}\text { Chromium, } \\
\text { dissolved } \\
(\mu \mathrm{g} / \mathrm{L})\end{array}$ & $\begin{array}{c}\text { Cobalt, } \\
\text { dissolved } \\
(\mu \mathrm{g} / \mathrm{L})\end{array}$ \\
\hline FC-BLANK & $9 / 17 / 2007$ & 1405 & ${ }^{1} 0.03$ & $<0.05$ & $<0.20$ & $<0.02$ & ${ }^{1} 0.04$ & $<0.02$ & ${ }^{1} 0.20$ \\
\hline FC-BLANK & $9 / 19 / 2007$ & 1425 & ${ }^{1} .04$ & .04 & $<.20$ & $<.02$ & $<.02$ & ${ }^{1} .06$ & ${ }^{1} .11$ \\
\hline FC-BLANK & $9 / 20 / 2007$ & 1205 & ${ }^{1} .02$ & $<.05$ & $<.10$ & $<.02$ & .01 & $<.02$ & ${ }^{1} .12$ \\
\hline FC-BLANK & $9 / 20 / 2007$ & 1305 & ${ }^{1} .03$ & $<.05$ & $<.10$ & $<.02$ & $<.01$ & $<.02$ & ${ }^{1} .26$ \\
\hline FC-BLANK & $9 / 21 / 2007$ & 1130 & ${ }^{1} .02$ & $<.05$ & $<.20$ & $<.02$ & $<.02$ & $<.02$ & ${ }^{1} .18$ \\
\hline
\end{tabular}

\begin{tabular}{|c|c|c|c|c|c|c|c|c|c|}
\hline Site ID & $\begin{array}{c}\text { Sample } \\
\text { extraction } \\
\text { date }\end{array}$ & $\begin{array}{c}\text { Time } \\
\text { (MDT) }\end{array}$ & $\begin{array}{l}\text { Copper, } \\
\text { dissolved } \\
(\mu \mathrm{g} / \mathrm{L})\end{array}$ & $\begin{array}{c}\text { Iron, } \\
\text { dissolved } \\
\text { ( } \mu \mathrm{g} / \mathrm{L})\end{array}$ & $\begin{array}{c}\text { Lead, } \\
\text { dissolved } \\
(\mu \mathrm{g} / \mathrm{L})\end{array}$ & $\begin{array}{c}\text { Manganese, } \\
\text { dissolved } \\
\text { ( } \mu \mathrm{g} / \mathrm{L})\end{array}$ & $\begin{array}{l}\text { Molybdenum, } \\
\text { dissolved } \\
(\mu \mathrm{g} / \mathrm{L})\end{array}$ & $\begin{array}{c}\text { Nickel, } \\
\text { dissolved } \\
(\mu \mathrm{g} / \mathrm{L})\end{array}$ & $\begin{array}{l}\text { Selenium, } \\
\text { dissolved } \\
\text { ( } \mu \mathrm{g} / \mathrm{L})\end{array}$ \\
\hline FC-BLANK & $9 / 17 / 2007$ & 1405 & $<0.30$ & $<3.0$ & $<0.07$ & ${ }^{1} 0.43$ & $<0.05$ & $<0.06$ & $<0.13$ \\
\hline FC-BLANK & $9 / 19 / 2007$ & 1425 & $<.30$ & $<3.0$ & $<.07$ & ${ }^{1} .25$ & ${ }^{1} .07$ & $<.06$ & $<.13$ \\
\hline FC-BLANK & $9 / 20 / 2007$ & 1205 & $<.30$ & ${ }^{1} 5.0$ & $<.07$ & $<.20$ & $<.05$ & $<.06$ & $<.13$ \\
\hline FC-BLANK & $9 / 20 / 2007$ & 1305 & $<.30$ & $<3.0$ & $<.07$ & ${ }^{1} .54$ & $<.05$ & $<.06$ & $<.13$ \\
\hline FC-BLANK & $9 / 21 / 2007$ & 1130 & ${ }^{1} .98$ & $<3.0$ & $<.07$ & ${ }^{1} .35$ & $<.05$ & $<.06$ & $<.13$ \\
\hline
\end{tabular}

\begin{tabular}{|c|c|c|c|c|c|c|c|}
\hline Site ID & $\begin{array}{c}\text { Sample } \\
\text { extraction } \\
\text { date }\end{array}$ & $\begin{array}{c}\text { Time } \\
\text { (MDT) }\end{array}$ & $\begin{array}{c}\text { Silver, } \\
\text { dissolved } \\
(\mu \mathrm{g} / \mathrm{L})\end{array}$ & $\begin{array}{c}\text { Thallium, } \\
\text { dissolved } \\
\text { ( } \mu \mathrm{g} / \mathrm{L} \text { ) }\end{array}$ & $\begin{array}{c}\text { Uranium, } \\
\text { dissolved } \\
\text { ( } \mu \mathrm{g} / \mathrm{L})\end{array}$ & $\begin{array}{c}\text { Vanadium, } \\
\text { dissolved } \\
\text { ( } \mu \mathrm{g} / \mathrm{L})\end{array}$ & $\begin{array}{c}\text { Zinc, } \\
\text { dissolved } \\
(\mu \mathrm{g} / \mathrm{L})\end{array}$ \\
\hline FC-BLANK & $9 / 17 / 2007$ & 1405 & 0.004 & $<0.01$ & 0.04 & $<0.02$ & 0.37 \\
\hline FC-BLANK & $9 / 19 / 2007$ & 1425 & ${ }^{1} .008$ & $<.01$ & .04 & $<.02$ & $<.60$ \\
\hline FC-BLANK & $9 / 20 / 2007$ & 1205 & $<.004$ & $<.01$ & $<.04$ & $<.02$ & $<.60$ \\
\hline FC-BLANK & $9 / 20 / 2007$ & 1305 & $<.004$ & $<.01$ & $<.04$ & $<.02$ & $<.60$ \\
\hline FC-BLANK & $9 / 21 / 2007$ & 1130 & $<.004$ & $<.01$ & .04 & $<.02$ & $<.60$ \\
\hline
\end{tabular}

\footnotetext{
${ }^{1}$ Values exceed lower reporting limit; Sb, 0.005; Cd, 0.02; Cr, 0.02; Co, 0.04; Cu, 0.30; Fe, 3.0; Mn, 0.20; Mo, 0.05; Ag, 0.004
} 
exception of one blank sample $(5 \mu \mathrm{g} / \mathrm{L})$. Concentrations of $\mathrm{Mn}$ ranged from 0.25 to $0.54 \mu \mathrm{g} / \mathrm{L}$ with a median concentration of $0.39 \mu \mathrm{g} / \mathrm{L}$. All Mo concentrations were less than the lower reporting limit with the exception of one blank sample $(0.07$ $\mu \mathrm{g} / \mathrm{L})$. Concentrations of $\mathrm{Sb}$ ranged from 0.02 to $0.04 \mu \mathrm{g} / \mathrm{L}$ with a median concentration of $0.03 \mu \mathrm{g} / \mathrm{L}$. Blank sample values greater than the laboratory reporting limits were still well below the water-quality standard values, which are the criteria for determining potentially hazardous sites.

Results of chemical analysis for the five replicate and associated leachate samples are presented in table 3. Although most of the results for the replicate analyses were within \pm 20 percent, results for a few elements in samples from selected Red, White, and Fry Canyon sites varied by more than \pm 20 percent: FC-343 (Ba, Cr, Fe, U); FC-394 (Al, Ca, Cr, $\mathrm{Sb}$ ); FC-735 (Al, Fe, Pb); FC-771 (Ag, Cu, Fe, Mo, Na, Pb, $\mathrm{U})$, and FC-814 (Al, Fe, $\mathrm{Pb}$ ) (table 3). The large percentage of difference in samples may be due to variations of mineral material within a waste dump sample.

\section{Solid-Phase Sample Digestion}

Fifteen of the 45 uranium waste dump samples were analyzed by the U.S. Geological Survey Geologic Division Laboratory in Denver, Colorado, for total extractible concentration. Forty-two elements (Ag, Al, As, Ba, Be, Bi, $\mathrm{Ca}, \mathrm{Cd}, \mathrm{Ce}, \mathrm{Co}, \mathrm{Cr}, \mathrm{Cs}, \mathrm{Cu}, \mathrm{Fe}, \mathrm{Ga}, \mathrm{In}, \mathrm{K}, \mathrm{La}, \mathrm{Li}, \mathrm{Mg}, \mathrm{Mn}$, Mo, Na, Nb, Ni, P, Pb, Rb, S, Sb, Sc, Sn, Sr, Te, Th, Ti, Tl, U, $\mathrm{V}, \mathrm{W}, \mathrm{Y}$, and $\mathrm{Zn}$ ) were analyzed by using inductively coupled plasma-atomic emission spectrometry (ICP-AES) following digestion of the solid sample with hydrochloric, nitric, perchloric, and hydrofluoric acids at low temperature (Crock and others, 1983). Analytical procedures and performance are presented in Briggs (2002). Selenium (Se) was analyzed by using hydride generation-atomic absorption spectrophotometry (HG-AAS) following digestion of the solid sample with nitric, hydrochloric, and sulfuric acids (Hageman and others, 2002). Mercury (Hg) was analyzed by using cold vapor-atomic absorption spectrometry (CV-AAS) following digestion of the solid sample with nitric acid and sodium dichromate (Brown and others, 2002).

\section{Assessment of Nonpoint Source Chemical Loading Potential}

\section{Leachate Results}

Trace-element concentrations in the 58 leachate samples collected from 45 uranium waste dump sites, 8 streambed sites, and 5 geologic background sites are shown in appendix A, Trace-element concentrations in the leachate samples from the uranium waste dump sites were compared with aquatic life (U.S. Environmental Protection Agency, 2007a) and drinking- water-quality standards (U.S. Environmental Protection Agency, 2007b) (table 4). Comparisons were not made for constituents without an aquatic life or drinking-water-quality standard. The proportions of leachate samples from uranium waste dump sites that exceeded drinking-water-quality standards were As 3/45, Be 2/45, Cd 2/45, Cu 10/45, Fe 3/45, Mn 25/45, U 21/45, and Zn 1/45. The ratios of leachate samples from uranium waste dump sites that exceeded aquatic life water-quality standards were $\mathrm{Cd} 18 / 45, \mathrm{Cu} 34 / 45, \mathrm{Ni}$ $10 / 45$, and $\mathrm{Zn} 16 / 45$.

Drinking-water-quality standards and trace-element concentrations for leachate samples are graphically presented using boxplots in figure 10. None of the constituents had median values that exceeded both the aquatic life and drinking-water-quality standards. However, mean values for $\mathrm{Cu}, \mathrm{Mn}$, and $\mathrm{U}$ exceeded drinking-water-quality standards. Approximately 71 percent (32/45) of the uranium waste dump leachate samples exceeded one or more drinking-water-quality standards. Mean values for $\mathrm{Cd}, \mathrm{Cu}$, and $\mathrm{Zn}$ exceeded aquatic life water-quality standards. Approximately 76 percent (34/45) of uranium waste dump leachate samples exceeded aquatic life water-quality standards.

Sites where concentrations of trace elements exceeded either or both aquatic life and drinking-water-quality standards are shown using bar graphs in figures 11-19. Aquatic life and (or) drinking-water-quality standards are shown for As (fig. 11), Be (fig. 12), $\mathrm{Cd}$ (fig. 13), $\mathrm{Cu}$ (fig. 14), $\mathrm{Fe}$ (fig. 15), $\mathrm{Mn}$ (fig. 16), $\mathrm{Ni}$ (fig. 17), U (fig. 18), and Zn (fig. 19). Several sample sites had one or more elements that exceeded both water-quality standards. Approximately 64 percent (29/45) of the uranium waste dump samples exceeded aquatic life and drinking-water-quality standards for one or more elements analyzed. The location of sample sites, site type, constituents that exceeded one or both water-quality standards, surrounding geology, and stream hydrology are shown in detail for map areas 1 through 4 (figs. 2-5).

Two or more elements exceeded drinking-water-quality standards at sites FC-318, FC-334, FC-394, FC-687, FC-709, FC-721, FC-725, FC-727, FC-758, FC-771, FC-772, FC-775, FC-784, FC-797, FC-800, FC-814, FC-819, FC-820, and FC-836. Three elements exceeded drinking-water-quality standards at sites FC-709, FC-721, FC-797, FC-800, FC-814, and FC-820; four elements exceeded drinking-water-quality standards at sites FC-772 and FC-775; and five elements exceeded drinking-water-quality standards at sites FC-318, FC-819, and FC-836.

Two or more elements exceeded aquatic life water-quality standards at sites FC-318, FC-324, FC-334, FC-394, FC-702, FC-709, FC-710, FC-721, FC-725, FC-758, FC-771, FC-772, FC-784, FC-797, FC-814, FC-819, FC-820, and FC-836. Three elements exceeded aquatic life water-quality standards at sites FC-721, FC-758, FC-771, FC-784, FC-814, and FC-820, and four elements exceeded aquatic life water-quality standards at sites FC-318, FC-324, FC-334, FC-709, FC-710, FC-725, FC-772, FC-797, FC-819, and FC-836.

Nearly all sites with samples that exceeded drinking-waterquality standards were uranium waste dump sites. However, 
Table 3. Chemical analysis of replicate and associated leachate samples from uranium waste dumps for selected major-ion and traceelement concentrations, Red, White, and Fry Canyons, southeastern Utah, 2007.

[Samples were analyzed at the Inductively Coupled Plasma-Mass Spectrometry Metals Analysis Laboratory at the University of Utah. Site ID, site identification; MDT, Mountain Daylight Time; DD.dddd, Degrees.decimal degrees; $\mu \mathrm{S} / \mathrm{cm}$, microsiemens per centimeter at 25 degrees Celsius; fil, filtered sample; lab, laboratory analysis; mg/L, milligrams per liter; $\mu \mathrm{g} / \mathrm{L}$, micrograms per liter; FC, Fry Canyon area; <, less than lower reporting limit; MI, matrix interference]

\begin{tabular}{|c|c|c|c|c|c|c|c|c|c|}
\hline Site ID & $\begin{array}{l}\text { Sample } \\
\text { extraction } \\
\text { date }\end{array}$ & $\begin{array}{c}\text { Time } \\
\text { (MDT) }\end{array}$ & $\begin{array}{l}\text { Latitude } \\
\text { (DD.dddd) }\end{array}$ & $\begin{array}{l}\text { Longitude } \\
\text { (DD.dddd) }\end{array}$ & $\begin{array}{c}\text { Altitude } \\
\text { (feet) }\end{array}$ & $\begin{array}{c}\text { pH } \\
\text { (standard } \\
\text { units) }\end{array}$ & $\begin{array}{c}\text { Specific } \\
\text { Conductance } \\
(\mu \mathrm{S} / \mathrm{cm})\end{array}$ & $\begin{array}{c}\text { Alkalinity, } \\
\text { fil, lab } \\
\text { (mg/L as } \\
\mathrm{CaCO}_{3} \text { ) }\end{array}$ & $\begin{array}{l}\text { Calcium, } \\
\text { dissolved } \\
\text { (mg/L) }\end{array}$ \\
\hline FC-343 & $9 / 20 / 2007$ & 1155 & 37.62942 & 110.10252 & 6,360 & 4.5 & 158 & MI & 22 \\
\hline FC-394 & $9 / 21 / 2007$ & 1120 & 37.56295 & 110.1024 & 6,200 & 4.4 & 169 & MI & ${ }^{1} 17$ \\
\hline FC-394 & $9 / 21 / 2007$ & 1125 & 37.56295 & 110.1024 & 6,200 & 4.3 & 139 & MI & ${ }^{1} 14$ \\
\hline FC-735 & $9 / 17 / 2007$ & 1505 & 37.54135 & 110.29038 & 4,820 & 6.8 & 496 & 5.0 & 76 \\
\hline FC-771 & $9 / 19 / 2007$ & 1420 & 37.5788 & 110.23853 & 5,280 & 5.6 & 453 & $<5.0$ & 59 \\
\hline FC-771 & $9 / 19 / 2007$ & 1415 & 37.5788 & 110.23853 & 5,280 & 5.6 & 468 & $<5.0$ & 65 \\
\hline FC-814 & $9 / 20 / 2007$ & 1255 & 37.57625 & 110.18958 & 5,720 & 5.8 & 204 & $<5.0$ & 18 \\
\hline FC-814 & $9 / 20 / 2007$ & 1300 & 37.57625 & 110.18958 & 5,720 & 5.6 & 217 & $<5.0$ & 19 \\
\hline
\end{tabular}

\begin{tabular}{|c|c|c|c|c|c|c|c|c|c|}
\hline Site ID & $\begin{array}{c}\text { Sample } \\
\text { extraction } \\
\text { date }\end{array}$ & $\begin{array}{l}\text { Magnesium, } \\
\text { dissolved } \\
\text { (mg/L) }\end{array}$ & $\begin{array}{l}\text { Potassium, } \\
\text { dissolved } \\
\text { (mg/L) }\end{array}$ & $\begin{array}{l}\text { Sodium, } \\
\text { dissolved } \\
\text { (mg/L) }\end{array}$ & $\begin{array}{c}\text { Sulfate, } \\
\text { dissolved } \\
\left(\mathrm{mg} / \mathrm{L} \text { as } \mathrm{SO}_{4}\right)\end{array}$ & $\begin{array}{l}\text { Aluminum, } \\
\text { dissolved } \\
(\mu \mathrm{g} / \mathrm{L})\end{array}$ & $\begin{array}{l}\text { Antimony, } \\
\text { dissolved } \\
(\mu \mathrm{g} / \mathrm{L})\end{array}$ & $\begin{array}{c}\text { Arsenic, } \\
\text { dissolved } \\
\text { ( } \mu \mathrm{g} / \mathrm{L})\end{array}$ & $\begin{array}{c}\text { Barium, } \\
\text { dissolved } \\
\text { ( } \mu \mathrm{g} / \mathrm{L})\end{array}$ \\
\hline FC-343 & $9 / 20 / 2007$ & 1.23 & 2.2 & 0.11 & 67 & 208 & 0.02 & 0.2 & ${ }^{1} 16$ \\
\hline FC-394 & $9 / 21 / 2007$ & 1.14 & 2.2 & .5 & 57 & ${ }^{1} 93$ & ${ }^{1} .09$ & 1.7 & 10 \\
\hline FC-394 & $9 / 21 / 2007$ & 0.95 & 2.1 & .43 & 48 & ${ }^{1} 70$ & ${ }^{1} .06$ & 1.8 & 9.4 \\
\hline FC-735 & $9 / 17 / 2007$ & 0.53 & 2.5 & .40 & 182 & ${ }^{1} 8.3$ & .09 & 1.3 & 24 \\
\hline FC-771 & $9 / 19 / 2007$ & 5.05 & 2.3 & ${ }^{1} .67$ & 171 & 31 & .10 & 1.6 & 9.9 \\
\hline FC-771 & $9 / 19 / 2007$ & 4.75 & 2.1 & ${ }^{1} .51$ & 190 & 34 & .11 & 1.8 & 8.5 \\
\hline FC-814 & $9 / 20 / 2007$ & 2.34 & 1.3 & 3.1 & 65 & ${ }^{1} 18$ & .16 & .4 & 7.8 \\
\hline FC-814 & $9 / 20 / 2007$ & 2.48 & 1.4 & 2.8 & 69 & ${ }^{1} 35$ & .17 & .5 & 8.6 \\
\hline
\end{tabular}

\begin{tabular}{|c|c|c|c|c|c|c|c|c|c|}
\hline Site ID & $\begin{array}{c}\text { Sample } \\
\text { extraction } \\
\text { date }\end{array}$ & $\begin{array}{l}\text { Cadmium, } \\
\text { dissolved } \\
(\mu \mathrm{g} / \mathrm{L})\end{array}$ & $\begin{array}{c}\text { Chromium, } \\
\text { dissolved } \\
(\mu \mathrm{g} / \mathrm{L})\end{array}$ & $\begin{array}{c}\text { Cobalt, } \\
\text { dissolved } \\
(\mu \mathrm{g} / \mathrm{L})\end{array}$ & $\begin{array}{c}\text { Copper, } \\
\text { dissolved } \\
(\mu \mathrm{g} / \mathrm{L})\end{array}$ & $\begin{array}{l}\text { Iron, } \\
\text { dissolved } \\
(\mu \mathrm{g} / \mathrm{L})\end{array}$ & $\begin{array}{l}\text { Lead, } \\
\text { dissolved } \\
(\mu \mathrm{g} / \mathrm{L})\end{array}$ & $\begin{array}{c}\text { Manganese, } \\
\text { dissolved } \\
(\mu \mathrm{g} / \mathrm{L})\end{array}$ & $\begin{array}{l}\text { Molybdenum, } \\
\text { dissolved } \\
(\mu \mathrm{g} / \mathrm{L})\end{array}$ \\
\hline FC-343 & $9 / 20 / 2007$ & 0.09 & ${ }^{1} 0.04$ & 15 & 6.1 & ${ }^{1} 9.0$ & ${ }^{1} 0.02$ & 164 & $<0.05$ \\
\hline FC-394 & $9 / 21 / 2007$ & .26 & ${ }^{1} .02$ & 23 & 1,000 & 6.0 & .03 & 74 & .65 \\
\hline FC-394 & $9 / 21 / 2007$ & .23 & ${ }^{1} .03$ & 19 & 840 & 6.0 & .03 & 64 & .60 \\
\hline FC-735 & $9 / 17 / 2007$ & $<.02$ & .10 & 0.88 & 22 & ${ }^{1} 24$ & ${ }^{1} .06$ & 7.0 & .18 \\
\hline FC-771 & $9 / 19 / 2007$ & .54 & .08 & 85 & ${ }^{1} 960$ & ${ }^{1} 15$ & ${ }^{1} .12$ & 144 & ${ }^{1} .13$ \\
\hline FC-771 & 9/19/2007 & .49 & .09 & 74 & ${ }^{1} 700$ & ${ }^{1} 42$ & ${ }^{1} .09$ & 130 & ${ }^{1} .16$ \\
\hline FC-814 & $9 / 20 / 2007$ & .49 & .05 & 23 & 3,100 & ${ }^{1} 4.0$ & $1<.07$ & 122 & .81 \\
\hline FC-814 & $9 / 20 / 2007$ & .52 & .05 & 25 & 3,300 & ${ }^{1} 16$ & ${ }^{1} .13$ & 127 & .71 \\
\hline
\end{tabular}


Table 3. Chemical analysis of replicate and associated leachate samples from uranium waste dumps for selected major-ion and traceelement concentrations, Red, White, and Fry Canyons, southeastern Utah, 2007.-Continued

[Samples were analyzed at the Inductively Coupled Plasma-Mass Spectrometry Metals Analysis Laboratory at the University of Utah. Site ID, site identification; MDT, Mountain Daylight Time; DD.dddd, Degrees.decimal degrees; $\mu \mathrm{S} / \mathrm{cm}$, microsiemens per centimeter at 25 degrees Celsius; fil, filtered sample; lab, laboratory analysis; mg/L, milligrams per liter; $\mu \mathrm{g} / \mathrm{L}$, micrograms per liter; FC, Fry Canyon area; <, less than lower reporting limit; MI, matrix interference]

\begin{tabular}{|c|c|c|c|c|c|c|c|c|}
\hline Site ID & $\begin{array}{c}\text { Sample } \\
\text { extraction } \\
\text { date }\end{array}$ & $\begin{array}{c}\text { Nickel, } \\
\text { dissolved } \\
(\mu \mathrm{g} / \mathrm{L})\end{array}$ & $\begin{array}{c}\text { Selenium, } \\
\text { dissolved } \\
(\mu \mathrm{g} / \mathrm{L})\end{array}$ & $\begin{array}{c}\text { Silver, } \\
\text { dissolved } \\
(\mu \mathrm{g} / \mathrm{L})\end{array}$ & $\begin{array}{c}\text { Thallium, } \\
\text { dissolved } \\
\text { ( } \mu \mathrm{g} / \mathrm{L})\end{array}$ & $\begin{array}{c}\text { Uranium, } \\
\text { dissolved } \\
(\mu \mathrm{g} / \mathrm{L})\end{array}$ & $\begin{array}{c}\text { Vanadium, } \\
\text { dissolved } \\
(\mu \mathrm{g} / \mathrm{L})\end{array}$ & $\begin{array}{c}\text { Zinc, } \\
\text { dissolved } \\
(\mu \mathrm{g} / \mathrm{L})\end{array}$ \\
\hline FC-343 & $9 / 20 / 2007$ & 14 & $<0.13$ & $<0.004$ & 0.11 & ${ }^{1} 3.8$ & 0.04 & 30 \\
\hline FC-343 & $9 / 20 / 2007$ & 12 & $<.13$ & $<.004$ & .11 & ${ }^{1} 2.9$ & .04 & 26 \\
\hline FC-394 & $9 / 21 / 2007$ & 16 & .18 & .02 & .17 & 46 & .07 & 71 \\
\hline FC-394 & $9 / 21 / 2007$ & 14 & .15 & .02 & .17 & 40 & .07 & 60 \\
\hline FC-735 & $9 / 17 / 2007$ & 0.70 & .11 & $<.004$ & .05 & 1.7 & .36 & 1.1 \\
\hline FC-735 & $9 / 17 / 2007$ & 0.71 & .12 & $<.004$ & .05 & 1.8 & .33 & 0.98 \\
\hline FC-771 & $9 / 19 / 2007$ & 46 & .33 & ${ }^{1} .03$ & .07 & ${ }^{1} 175$ & .21 & 275 \\
\hline FC-771 & 9/19/2007 & 40 & .32 & ${ }^{1} .01$ & .07 & ${ }^{1} 138$ & .24 & 241 \\
\hline FC-814 & $9 / 20 / 2007$ & 14 & $<.13$ & .02 & .06 & 140 & .11 & 145 \\
\hline FC-814 & $9 / 20 / 2007$ & 15 & $<.13$ & .02 & .06 & 139 & .12 & 153 \\
\hline
\end{tabular}

${ }^{1}$ Replicate samples exceeding \pm 20 percent

Table 4. U.S. Environmental Protection Agency drinking-waterquality standards and aquatic life water-quality standards.

[ $\mu \mathrm{g} / \mathrm{L}$, micrograms per liter; NA, not applicable]

\begin{tabular}{|c|c|c|}
\hline Element & $\begin{array}{c}\text { 'Drinking- } \\
\text { water standard } \\
\text { concentration ( } \mu \mathrm{g} / \mathrm{L})\end{array}$ & $\begin{array}{c}{ }^{2} \text { Aquatic life } \\
\text { water standard } \\
\text { concentration ( } \mu \mathrm{g} / \mathrm{L})\end{array}$ \\
\hline Antimony (Sb) & 6.0 & NA \\
\hline Arsenic (As) & 10 & 150 \\
\hline Barium $(\mathrm{Ba})$ & 2,000 & NA \\
\hline Beryllium (Be) & 4.0 & NA \\
\hline Cadmium (Cd) & 5.0 & ${ }^{3} 0.25$ \\
\hline Chromium (Cr) & 100 & NA \\
\hline Copper $(\mathrm{Cu})$ & 1,300 & ${ }^{3} 9.0$ \\
\hline Iron $(\mathrm{Fe})$ & 300 & NA \\
\hline Lead $(\mathrm{Pb})$ & 15 & ${ }^{3} 2.5$ \\
\hline Manganese (Mn) & 50 & NA \\
\hline Mercury (Hg) & 2.0 & 0.77 \\
\hline Molybdenum (Mo) & NA & NA \\
\hline Nickel (Ni) & NA & ${ }^{3} 52$ \\
\hline Selenium (Se) & 50 & 5.0 \\
\hline Silver (Ag) & 100 & NA \\
\hline Thallium (Tl) & 2.0 & NA \\
\hline Uranium (U) & 30 & NA \\
\hline Vanadium (V) & NA & NA \\
\hline Zinc $(\mathrm{Zn})$ & 5,000 & ${ }^{3} 120$ \\
\hline
\end{tabular}

${ }^{1}$ Drinking-water-quality standards (U.S. Environmental Protection Agency, 2007b)

${ }^{2}$ Aquatic life water-quality standards (U.S. Environmental Protection Agency, 2007a)

${ }^{3}$ Aquatic life standard is based on a hardness value of $100 \mu \mathrm{g} / \mathrm{L}$ two background samples and three streambed-sediment samples also exceeded water-quality standards. Iron and uranium concentrations exceeded the drinking-water-quality standard in the background sample from site FC-790-BG and arsenic concentrations exceeded the drinking-water-quality standard and copper concentrations exceeded the aquatic life water-quality standard in the background sample from site FC-682-BG. Iron concentrations exceeded drinking-waterquality standards at streambed-sediment sites FC-334-SED, FC-814-SED, and FC-836-SED, and manganese concentrations exceeded the drinking water-quality standard from site FC-710-SED. Standards were not exceeded in samples from other background or streambed sites. Most uranium waste dump sites are located in or near ephemeral streams that would likely minimize their contaminant contribution to perennial streams. Select trace element concentrations for all samples exceeded water-quality standards for both drinking water and aquatic life at sites FC-305, FC-318, FC-709, FC-721, FC-772, FC-775, FC-797, FC-814, FC-819, FC-820, and FC-836, located in Red Canyon. Dissolved copper concentrations were largest in samples from sites FC-721, FC-772, FC-797, FC-814, FC-819, FC-820, and FC-836, ranging from 3,300 $\mu \mathrm{g} / \mathrm{L}$ (FC-814) to $92,000 \mu \mathrm{g} / \mathrm{L}$ (FC-772) (fig. 14). Dissolved uranium concentrations were largest in samples from sites FC-772 and FC-819, ranging from $990 \mu \mathrm{g} / \mathrm{L}$ (FC-819) to $1,200 \mu \mathrm{g} / \mathrm{L}$ (FC-772) (fig. 18). 

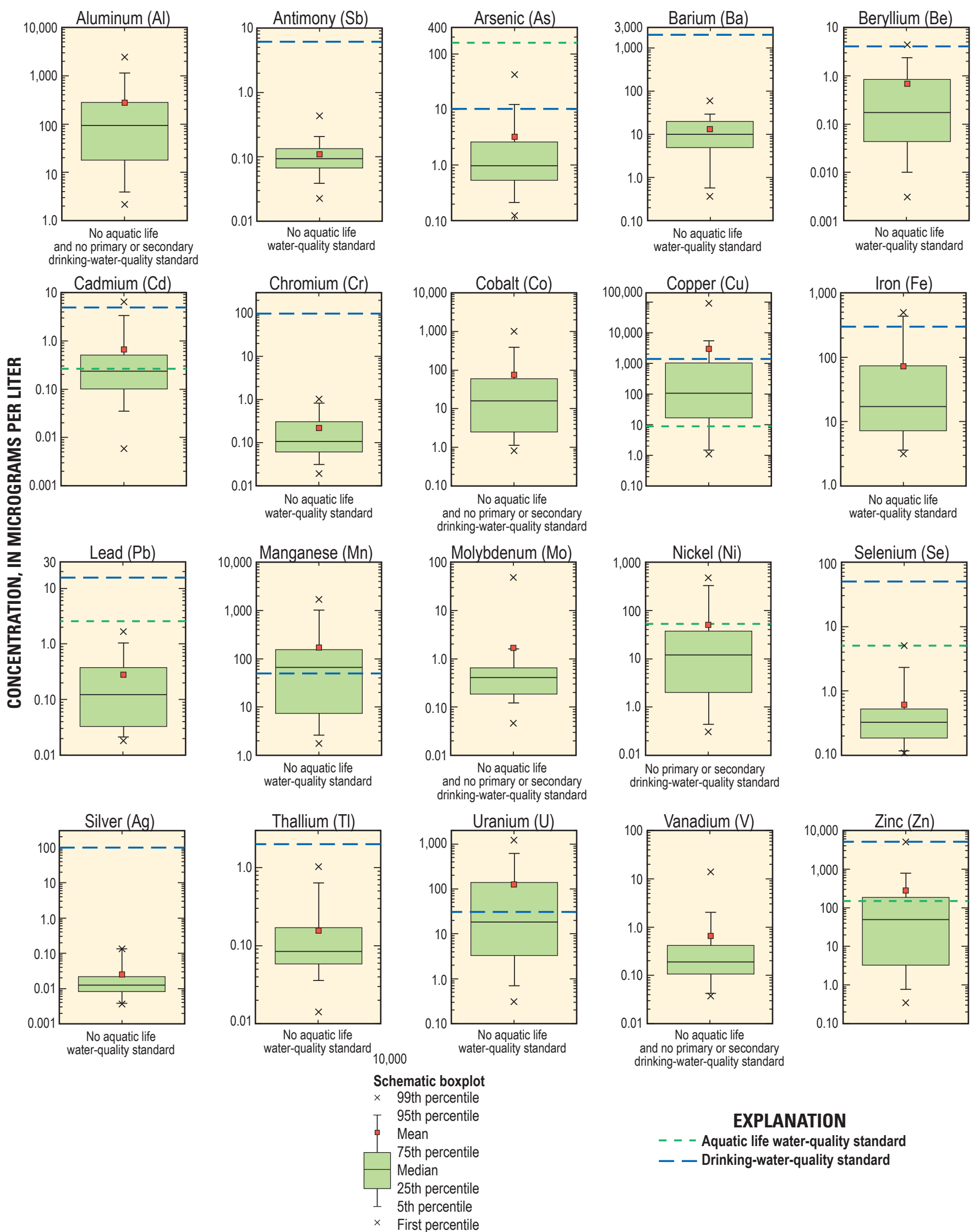

\section{EXPLANATION}

- - - Aquatic life water-quality standard

_ D Drinking-water-quality standard

Figure 10. Concentrations of dissolved trace elements in leachate extractions from the 45 uranium waste dump samples collected from Red, White, and Fry Canyons, southeastern Utah, 2007. 


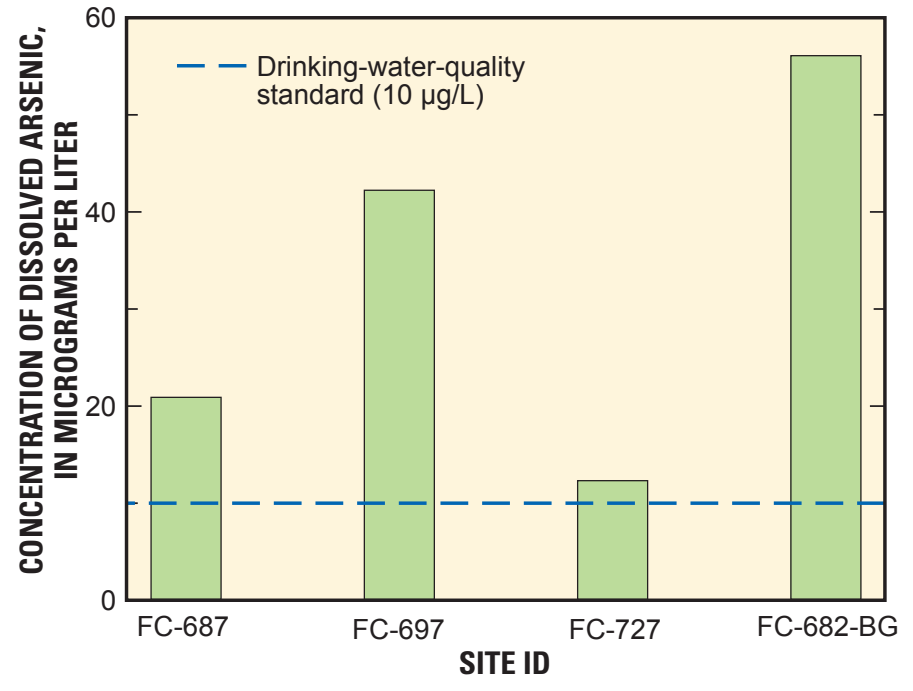

Figure 11. Concentrations of dissolved arsenic in uranium waste dump leachate samples that exceeded aquatic life and drinking-water-quality standards, Red, White, and Fry Canyons, southeastern Utah, 2007.

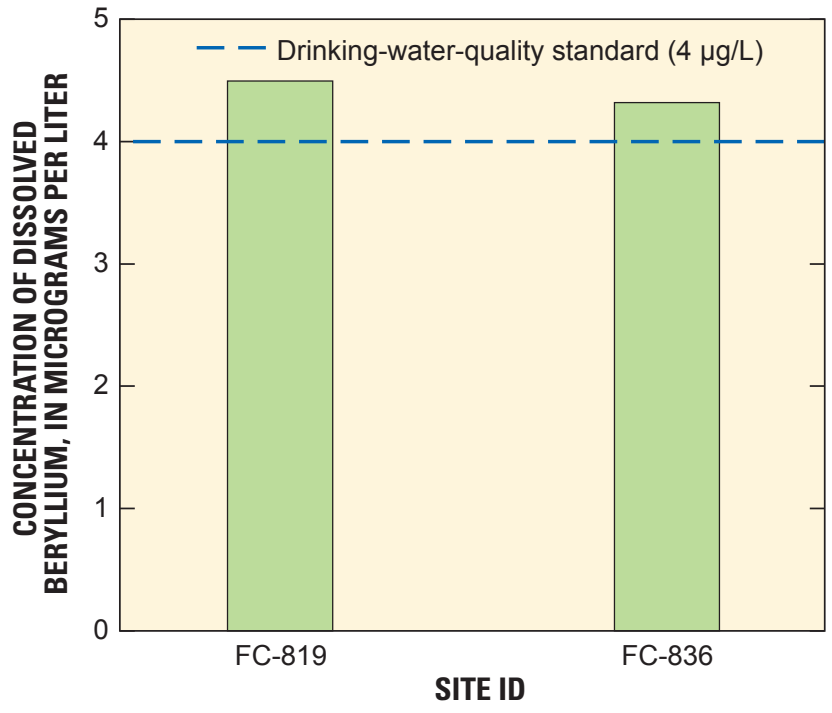

Figure 12. Concentrations of dissolved beryllium in uranium waste dump leachate samples that exceeded drinking-waterquality standards, Red, White, and Fry Canyons, southeastern Utah, 2007.

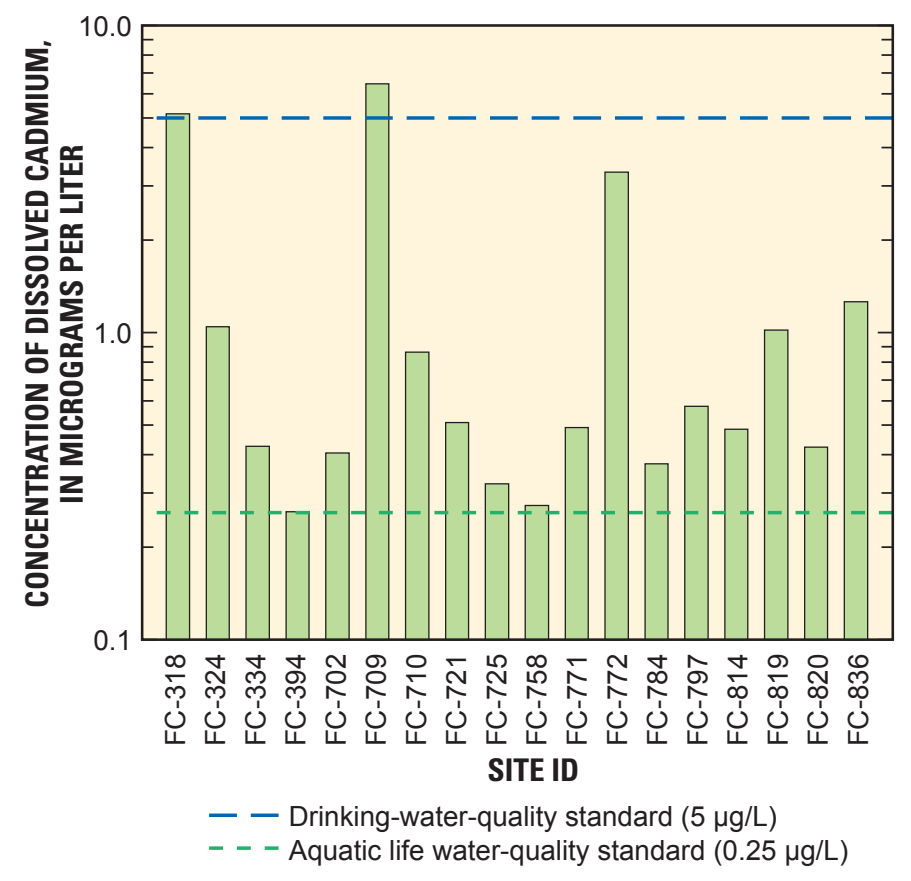

Figure 13. Concentrations of dissolved cadmium in uranium waste dump leachate samples that exceeded aquatic life and drinkingwater-quality standards, Red, White, and Fry Canyons, southeastern Utah, 2007. 


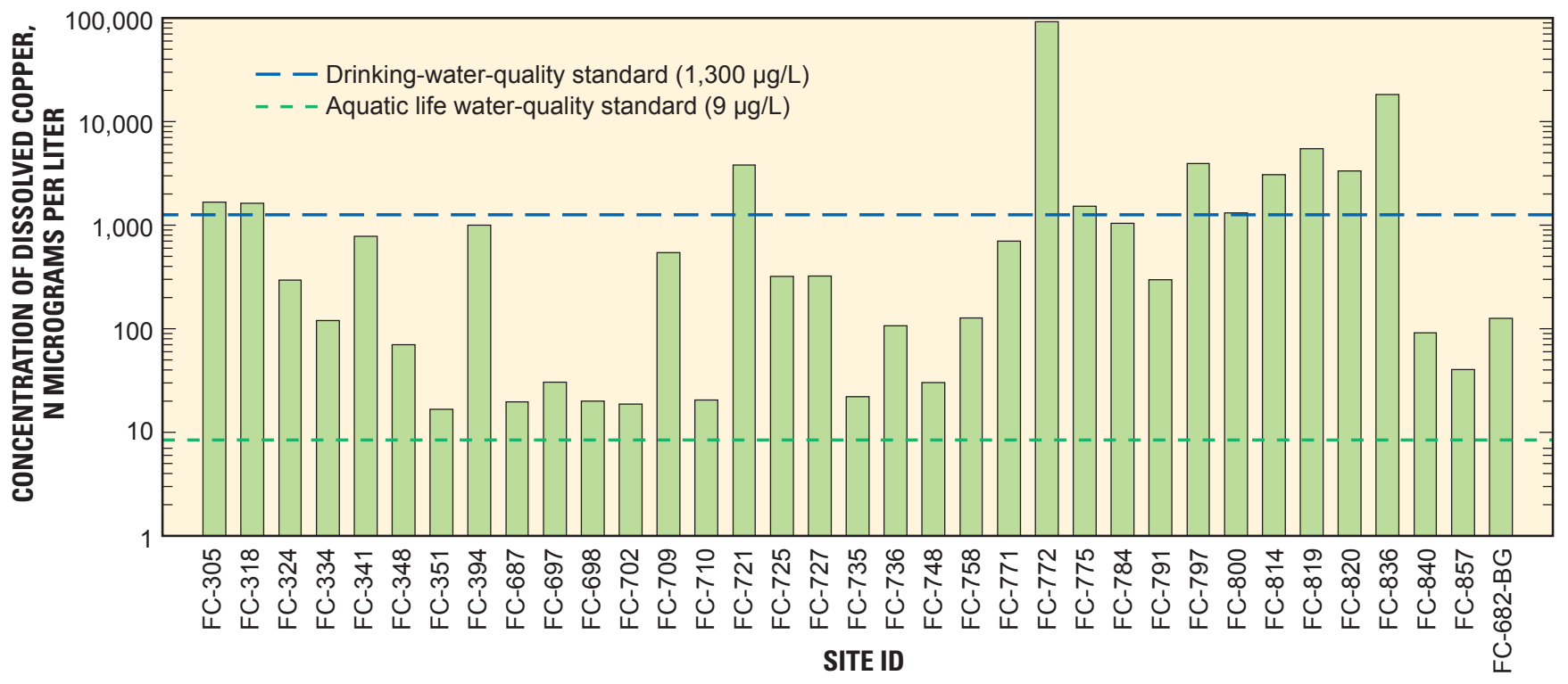

Figure 14. Concentrations of dissolved copper in uranium waste dump leachate samples that exceeded aquatic life and drinkingwater-quality standards, Red, White, and Fry Canyons, southeastern Utah, 2007.

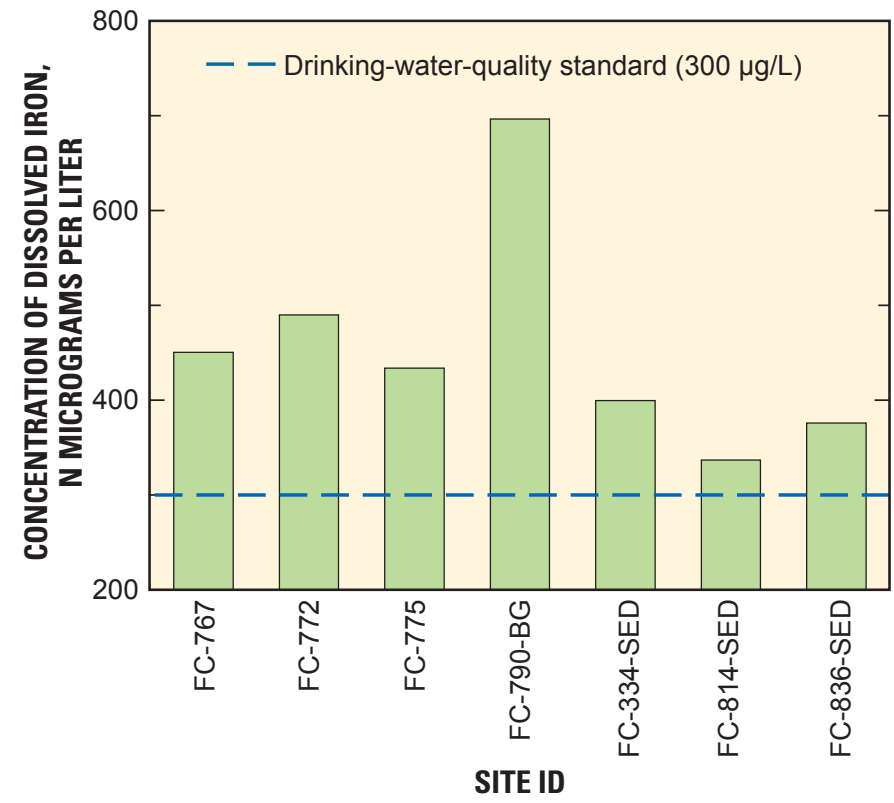

Figure 15. Concentrations of dissolved iron in uranium waste dump leachate samples that exceeded drinking-water-quality standards, Red, White, and Fry Canyons, southeastern Utah, 2007. 


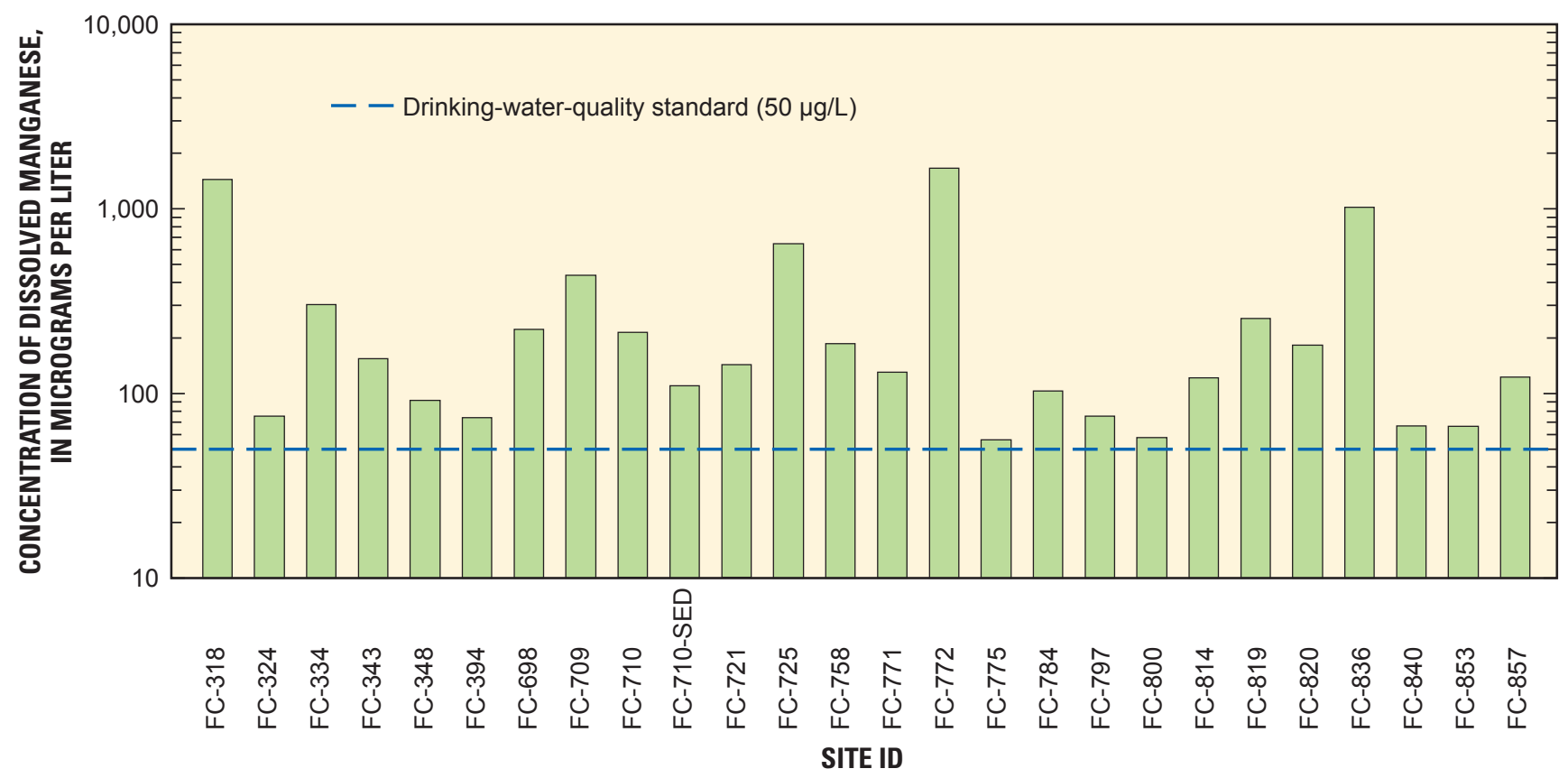

Figure 16. Concentrations of dissolved manganese in uranium waste dump leachate samples that exceeded drinking-water-quality standards, Red, White, and Fry Canyons, southeastern Utah, 2007.

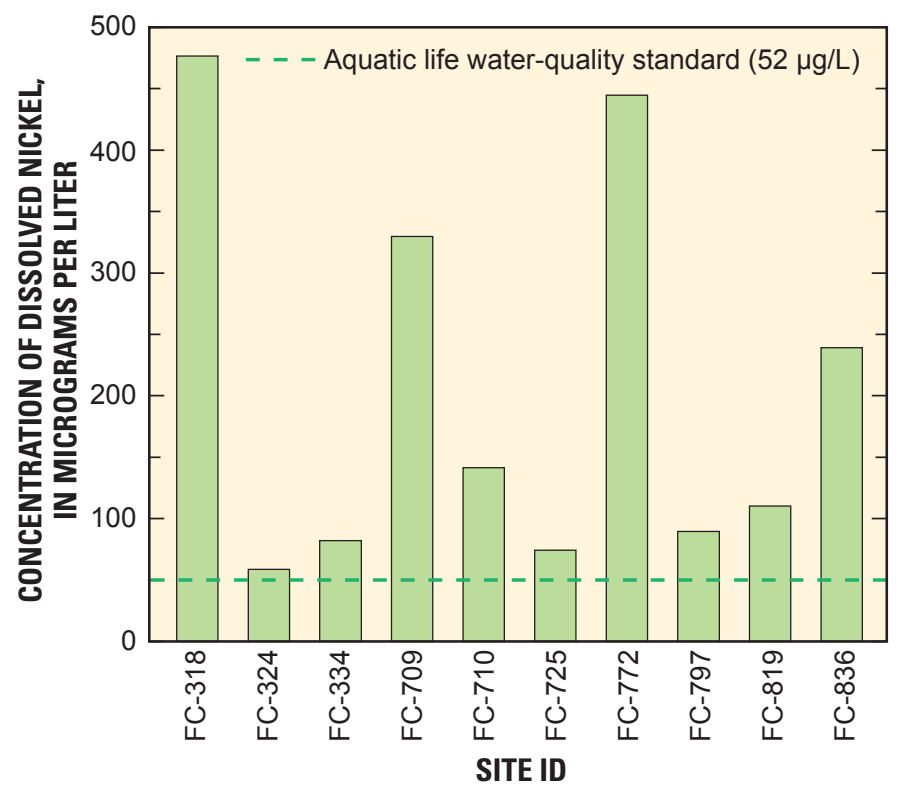

Figure 17. Concentrations of dissolved nickel in uranium waste dump leachate samples that exceeded aquatic life water-quality standards, Red, White, and Fry Canyons, southeastern Utah, 2007. 


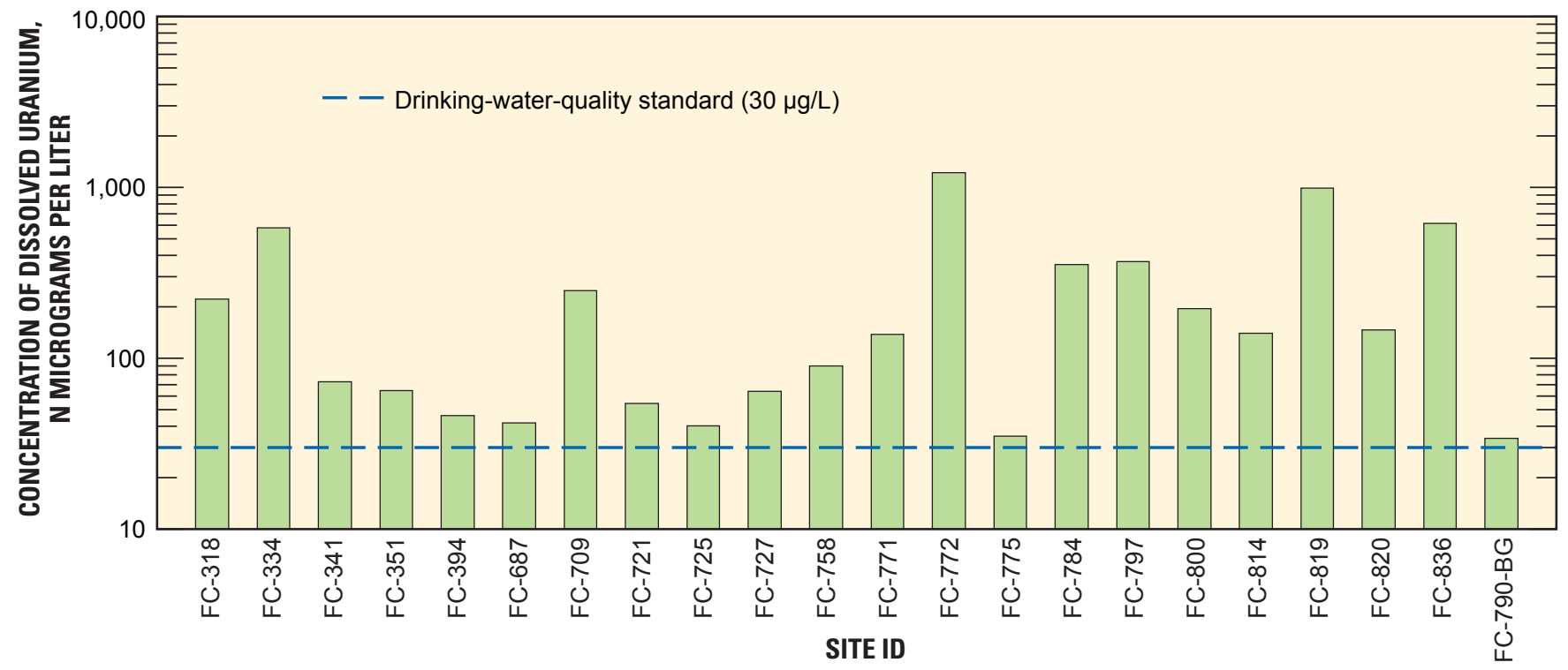

Figure 18. Concentrations of dissolved uranium in uranium waste dump leachate samples that exceeded drinking-water-quality standards, Red, White, and Fry Canyons, southeastern Utah, 2007.

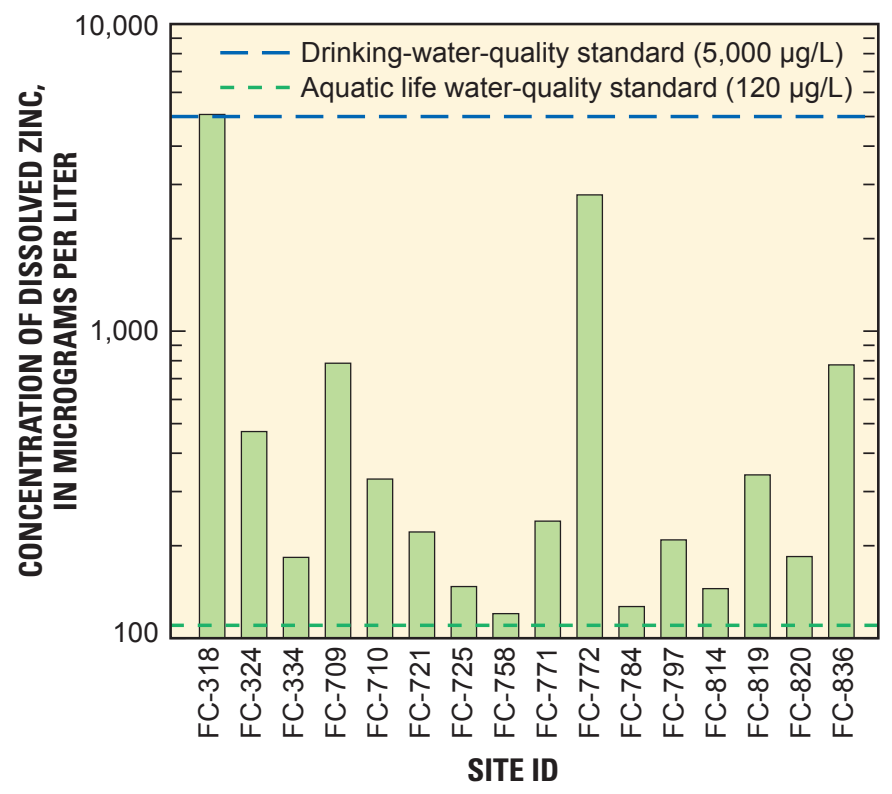

Figure 19. Concentrations of dissolved zinc in uranium waste dump leachate samples that exceeded drinking-water-quality standards, Red, White, and Fry Canyons, southeastern Utah, 2007.

\section{StreamStats Analysis of Waste Dump Sites}

Periodic rainfall events may fill ephemeral stream drainages with water and transport some of the waste dump material off site. Because of the episodic nature of streamflow in the Red, White, and Fry Canyons, no permanent gaging stations are currently recording flow. A set of multiple-linear regression equations have been developed for the State of Utah to estimate peak flows in ungaged drainages. All uranium waste dump sites in Red, White, and Fry Canyons are located within EPA Region 6, and regression equations for 2-, 100-, and 500-year recurrence interval peak flows are given below (Kenney and others, 2007):

$$
\begin{aligned}
& \text { PK2 }=4,150 \text { DRNAREA }^{0.553}\left({\text { ELEV } / 1,000)^{-2.45}}^{-2}\right. \\
& \text { PK100 }=115,000 \text { DRNAREA }^{0.391}(\text { ELEV/1,000 })^{-2.58} \\
& \text { PK500 }=258,000 \text { DRNAREA }{ }^{0.344}(\text { ELEV/1,000 })^{-2.65}
\end{aligned}
$$

where,

PK is the peak flow; number following PK represents recurrence interval, in years,

DRNAREA is the drainage area, in square miles, and ELEV is the mean basin elevation, in feet.

The parameters and equations for drainage areas throughout Utah were made into an interactive website, StreamStats (Ries and others, 2004). All sample sites were input into the USGS Utah StreamStats website at http://streamstatsags.cr.usgs.gov/ ut_ss/default.aspx?stabbr $=u t \& d t=1241905018973$ to determine the drainage basin characteristics contributing to runoff at the uranium waste dump sites (table 5). Four uranium waste dump sites with the largest drainage basin areas on the basis of the StreamStats delineation were FC-394 (2.8 mi²), 
Table 5. Drainage area characteristics associated with uranium waste dump samples, Red, White, Blue Notch, and Fry Canyons, southeastern Utah.

[Site ID, site identification; DD.dddd, Degrees.decimal degrees; PK 2, 2-year peak flow; PK 100, 100-year peak flow; PK 500, 500-year peak flow; FC, Fry Canyon area; BG, background; SED, sediment; NA, not applicable]

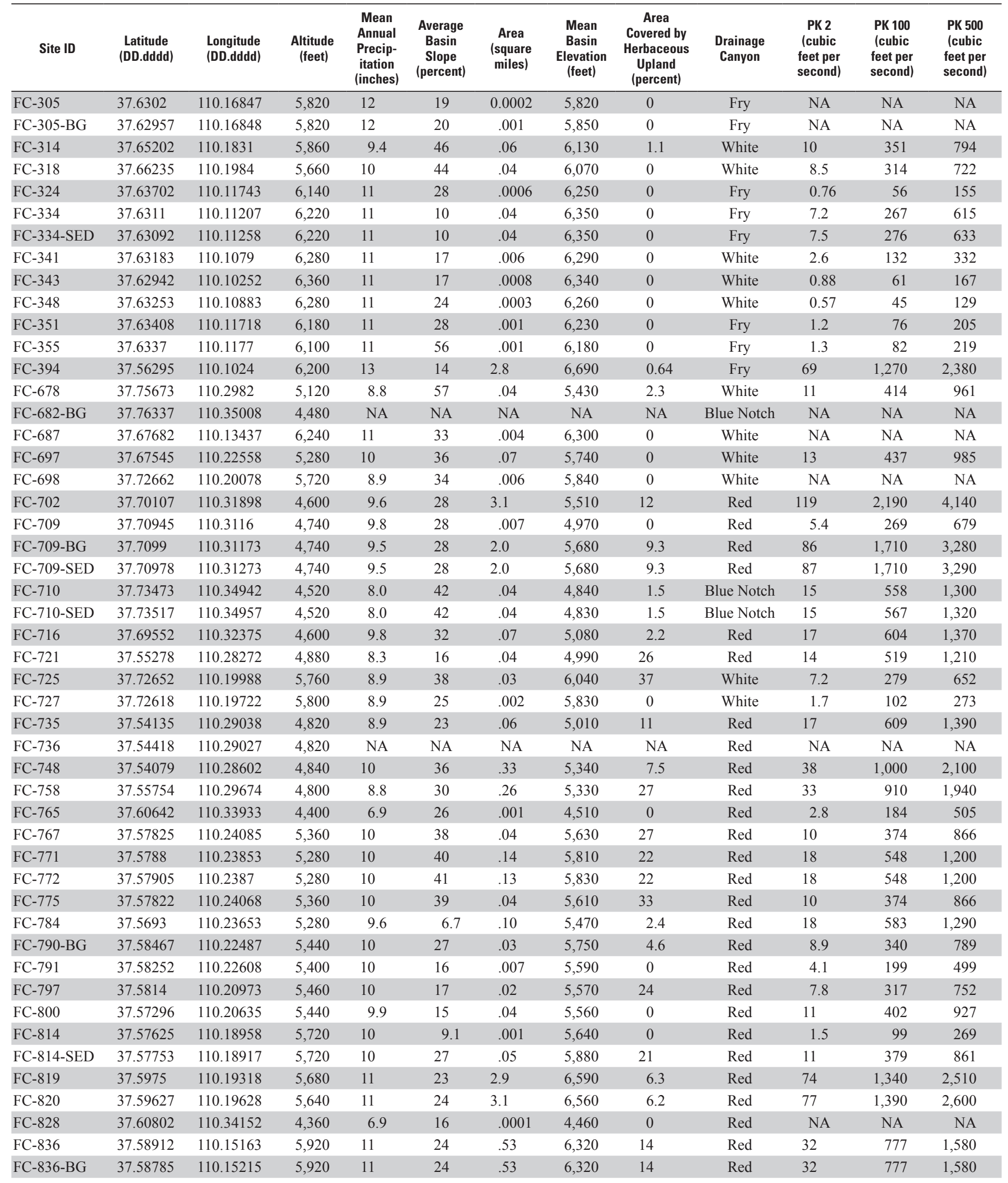


Table 5. Drainage area characteristics associated with uranium waste dump samples, Red, White, Blue Notch, and Fry Canyons, southeastern Utah.-Continued

[Site ID, site identification; DD.dddd, Degrees.decimal degrees; PK 2, 2-year peak flow; PK 100, 100-year peak flow; PK 500, 500-year peak flow; FC, Fry Canyon area; BG, background; SED, sediment; NA, not applicable]

\begin{tabular}{|c|c|c|c|c|c|c|c|c|c|c|c|c|}
\hline Site ID & $\begin{array}{l}\text { Latitude } \\
\text { (DD.dddd) }\end{array}$ & $\begin{array}{l}\text { Longitude } \\
\text { (DD.dddd) }\end{array}$ & $\begin{array}{c}\text { Altitude } \\
\text { (feet) }\end{array}$ & $\begin{array}{l}\text { Mean } \\
\text { Annual } \\
\text { Precip- } \\
\text { itation } \\
\text { (inches) }\end{array}$ & $\begin{array}{l}\text { Average } \\
\text { Basin } \\
\text { Slope } \\
\text { (percent) }\end{array}$ & $\begin{array}{c}\text { Area } \\
\text { (square } \\
\text { miles) }\end{array}$ & $\begin{array}{c}\text { Mean } \\
\text { Basin } \\
\text { Elevation } \\
\text { (feet) }\end{array}$ & $\begin{array}{c}\text { Area } \\
\text { Covered by } \\
\text { Herbaceous } \\
\text { Upland } \\
\text { (percent) }\end{array}$ & $\begin{array}{c}\text { Drainage } \\
\text { Canyon }\end{array}$ & $\begin{array}{c}\text { PK 2 } \\
\text { (cubic } \\
\text { feet per } \\
\text { second) }\end{array}$ & $\begin{array}{l}\text { PK } 100 \\
\text { (cubic } \\
\text { feet per } \\
\text { second) }\end{array}$ & $\begin{array}{c}\text { PK } 500 \\
\text { (cubic } \\
\text { feet per } \\
\text { second) }\end{array}$ \\
\hline FC-836-SED & 37.58788 & 110.15255 & 5,920 & 11 & 24 & 0.53 & 6,320 & 14 & Red & 32 & 777 & 1,580 \\
\hline FC-840 & 37.5846 & 110.12691 & 6,000 & 11 & 31 & .14 & 6,330 & 1.1 & Fry & 15 & 456 & 987 \\
\hline FC-840-SED & 37.58537 & 110.12185 & 6,000 & 11 & 30 & .21 & 6,190 & 1.9 & Fry & 20 & 566 & 1,200 \\
\hline FC-853 & 37.76457 & 110.36922 & 4,280 & 8.1 & 37 & .49 & 4,890 & 15 & Blue Notch & 57 & 1,450 & 3,010 \\
\hline FC-857 & 37.54968 & 110.30207 & 4,800 & 8.8 & 34 & .21 & 5,190 & 7.0 & Red & 31 & 892 & 1,920 \\
\hline FC-857-SED & 37.5515 & 110.30043 & 4,800 & 8.8 & 32 & .27 & 5,070 & 6.3 & Red & 38 & 1,050 & 2,230 \\
\hline FC-866 & 37.5492 & 110.30237 & 4,760 & 8.8 & 34 & .21 & 5,190 & 6.6 & Red & 31 & 892 & 1,920 \\
\hline FC-885 & 37.5879 & 110.12867 & 5,980 & 11 & 19 & .0002 & 5,940 & 0 & Fry & 0.52 & 44 & 129 \\
\hline FC-885-SED & 37.59027 & 110.12382 & 5,980 & 11 & 24 & .04 & 5,750 & 0 & Fry & 10 & 374 & 859 \\
\hline
\end{tabular}

FC-702 (3.1 mi²), FC-819 $\left(2.9 \mathrm{mi}^{2}\right)$, and FC-820 $\left(3.1 \mathrm{mi}^{2}\right)$. Six uranium waste dump sites with steep average basin slopes (more than 40 percent) were FC-314, FC-318, FC-355, FC-678, FC-710, and FC-772. Average basin slopes for these sites were $46,44,56,57,42$, and 41 percent, respectively.

The valid range for the regression equations (1-3) is a drainage area between 0.87 and $532 \mathrm{mi}^{2}$ and a mean basin elevation between 4,300 and 9,380 ft. Many drainage area values were less than the minimum value of $0.87 \mathrm{mi}^{2}$, but all mean basin elevation values fell within the valid range (table 5). Peak flows estimated from values outside of valid regression equation ranges are extrapolations with unknown errors. The errors given for values within the valid range for PK2, PK100, and PK500 are 110, 61, and 66 percent average standard error of prediction and $1.44,13$, and 15 equivalent years of record, respectively (Kenney and others, 2007).

\section{Human Health Hazard Assessment}

Fifteen uranium waste dump samples were analyzed for total digestible metal concentration (table 6). Samples were analyzed for major ( $\mathrm{Ca}, \mathrm{K}, \mathrm{Mg}, \mathrm{Na}, \mathrm{P}, \mathrm{S})$, trace (Ag, Al, As, $\mathrm{Ba}, \mathrm{Be}, \mathrm{Bi}, \mathrm{Cd}, \mathrm{Co}, \mathrm{Cr}, \mathrm{Cs}, \mathrm{Cu}, \mathrm{Fe}, \mathrm{Ga}, \mathrm{Hg}, \mathrm{In}, \mathrm{Li}, \mathrm{Mn}, \mathrm{Mo}$, $\mathrm{Nb}, \mathrm{Ni}, \mathrm{Pb}, \mathrm{Rb}, \mathrm{Sb}, \mathrm{Sc}, \mathrm{Se}, \mathrm{Sn}, \mathrm{Sr}, \mathrm{Te}, \mathrm{Th}, \mathrm{Ti}, \mathrm{Tl}, \mathrm{U}, \mathrm{V}, \mathrm{W}, \mathrm{Y}$, $\mathrm{Zn})$, and rare earth elements (Ce, La).

A relation between leachate and total digestible uranium concentration has been determined based on a regression equation with all known data (fig. 20):

$$
\mathrm{U}_{\text {total }(\mathrm{ppm})}=28.434 \ln \left(\mathrm{U}_{\text {leachate }(\mu \mathrm{g} / \mathrm{L})}\right)+59.093
$$

Equation 4 was used to determine the total concentration for the uranium waste dump sites with only a leachate analysis (table 7). Data from waste dump site FC-721 was not used to compute the regression equation because it was determined to be an outlier.
Three soil screening levels (SSL) for various exposure durations were determined for the combined carcinogenic effects of uranium and radium as radionuclides based on calculations made with RESRAD 6.5, a computer model designed to estimate radiation doses and risks from residual radioactive materials (Yu and others, 1993). Soil screening levels were determined with respect to a probable concentration of radium and all other uranium decay series radionuclides set to secular equilibrium with radium-226. In the Colorado Plateau region uranium deposits, secular equilibrium between radium and uranium is typically not observed, and in many places, uranium deposits are depleted in radium with respect to uranium by daughter products leached from primary uranium minerals by forming secondary minerals from changing groundwater conditions and oxidation (Stern and Stieff, 1959). Since the sites in this study were not sampled for radium, a concentration range for radium was considered. The equilibrium radium concentration, or high end concentration, assumes that radium is in secular equilibrium with uranium in any waste dump pile. The leached concentration, or low end concentration, assumes that radium activities are approximately 57 percent of uranium activities in any waste dump pile. The radium-uranium ratio of 0.57 is derived from a similar study conducted in Brown's Hole, Utah, where sites were sampled for radium and uranium (Tom Marston, U.S. Geological Survey, written commun. 2010).

Soil screening levels were evaluated for a maximum annual radiation dosage of 15 mrem per year (U.S. Environmental Protection Agency, 1997a). Several exposure pathways were considered in the model: direct external exposure to contaminated soils, internal exposure to inhaled dust, and internal exposure by ingestion of contaminated soils. RESRAD also considers exposures from ingested plants grown in contaminated soils, from ingested meat derived from livestock fed with contaminated feed or water, from drinking water directly, and from ingested fish from a contaminated body of water. These pathways were not included based on 
Table 6. Chemical analysis of total extractible from samples from uranium waste dumps for selected major-ion and trace-element concentrations, Red, White, and Fry Canyons, southeastern Utah, 2007.

[Samples were analyzed at the U.S. Geological Survey Geologic Discipline Laboratory, Denver, Colorado. Site ID, site identification; MDT, Mountain Daylight Time; DD.dddd, Degrees.decimal degrees; \%, percent; ppm, parts per million; FC, Fry Canyon area; <, less than lower reporting limit]

\begin{tabular}{|c|c|c|c|c|c|c|c|c|c|c|c|c|c|}
\hline Site ID & $\begin{array}{c}\text { Sample } \\
\text { extraction } \\
\text { date }\end{array}$ & $\begin{array}{c}\text { Time } \\
\text { (MDT) }\end{array}$ & $\begin{array}{l}\text { Latitude } \\
\text { (DD.dddd) }\end{array}$ & \multicolumn{2}{|c|}{$\begin{array}{l}\text { Longitude } \\
\text { (DD.dddd) }\end{array}$} & $\begin{array}{l}\text { Altitude } \\
\text { (feet) }\end{array}$ & $\begin{array}{l}\text { Aluminum, } \\
\text { total } \\
(\%)\end{array}$ & $\begin{array}{c}\text { Antimony, } \\
\text { total } \\
\text { (ppm) }\end{array}$ & $\begin{array}{c}\text { Arsenic, } \\
\text { total } \\
(\mathrm{ppm})\end{array}$ & $\begin{array}{c}\text { Barium, } \\
\text { total } \\
\text { (ppm) }\end{array}$ & $\begin{array}{l}\text { Beryllium, } \\
\text { total } \\
\text { (ppm) }\end{array}$ & $\begin{array}{c}\text { Bismuth, } \\
\text { total } \\
\text { (ppm) }\end{array}$ & $\begin{array}{c}\text { Cadmium, } \\
\text { total } \\
\text { (ppm) }\end{array}$ \\
\hline FC-318 & $9 / 21 / 2007$ & 1045 & 37.66235 & \multicolumn{2}{|c|}{110.1984} & 5,660 & 4.5 & 1.3 & 74 & 240 & 2.3 & 0.17 & 0.50 \\
\hline FC-334 & $9 / 20 / 2007$ & 1240 & 37.6311 & \multicolumn{2}{|c|}{110.11207} & 6,220 & 3.6 & 1.7 & 110 & 240 & 1.1 & .15 & $<.10$ \\
\hline FC-343 & $9 / 20 / 2007$ & 1155 & 37.62942 & \multicolumn{2}{|c|}{110.10252} & 6,360 & 6.4 & 0.63 & 45 & 240 & 2.2 & .23 & $<.10$ \\
\hline FC-355 & $9 / 20 / 2007$ & 1225 & 37.6337 & \multicolumn{2}{|c|}{110.1177} & 6,100 & 3.6 & 1.6 & 120 & 480 & 1.5 & $<.04$ & .30 \\
\hline FC-721 & 9/19/2007 & 1435 & 37.55278 & \multicolumn{2}{|c|}{110.28272} & 4,880 & 6.7 & 1.7 & 97 & 730 & 3.4 & .09 & .20 \\
\hline FC-765 & $9 / 21 / 2007$ & 1105 & 37.60642 & \multicolumn{2}{|c|}{110.33933} & 4,400 & 2.6 & 0.44 & 25 & 320 & 0.80 & .11 & .10 \\
\hline FC-771 & 9/19/2007 & 1415 & 37.5788 & \multicolumn{2}{|c|}{110.23853} & 5,280 & 5.9 & 1.0 & 100 & 360 & 3.5 & .19 & .20 \\
\hline FC-772 & $9 / 20 / 2007$ & 1245 & 37.57905 & \multicolumn{2}{|c|}{110.2387} & 5,280 & 3.3 & 0.97 & 71 & 380 & 1.5 & .14 & .30 \\
\hline FC-775 & 9/20/2007 & 1145 & 37.57822 & \multicolumn{2}{|c|}{110.24068} & 5,360 & 5.9 & 1.4 & 78 & 320 & 3.2 & .20 & .10 \\
\hline FC-853 & $9 / 21 / 2007$ & 1040 & 37.76457 & 110 & 6922 & 4,280 & 2.4 & 1.1 & 230 & 550 & 1.1 & .38 & .30 \\
\hline Site ID & $\begin{array}{c}\text { Calcium, } \\
\text { total } \\
(\%)\end{array}$ & $\begin{array}{l}\text { Cerium, } \\
\text { total } \\
\text { (ppm) }\end{array}$ & $\begin{array}{c}\text { Cesium, Ch } \\
\text { total } \\
\text { (ppm) }\end{array}$ & $\begin{array}{c}\text { Chromium, } \\
\text { total } \\
(\mathrm{ppm})\end{array}$ & $\begin{array}{c}\text { Cobalt, } \\
\text { total } \\
\text { (ppm) }\end{array}$ & $\begin{array}{c}\text { Copper, } \\
\text { total } \\
\text { (ppm) }\end{array}$ & $\begin{array}{c}\text { Gallium, } \\
\text { total } \\
\text { (ppm) }\end{array}$ & $\begin{array}{l}\text { Indium, } \\
\text { total } \\
\text { (ppm) }\end{array}$ & $\begin{array}{l}\text { Iron, } \\
\text { total } \\
(\%)\end{array}$ & $\begin{array}{c}\text { Lanthanum, } \\
\text { total } \\
\text { (ppm) }\end{array}$ & $\begin{array}{l}\text { Lithium, } \\
\text { total } \\
\text { (ppm) }\end{array}$ & $\begin{array}{l}\text { Lead, } \\
\text { total } \\
(\mathrm{ppm})\end{array}$ & $\begin{array}{l}\text { Magnesium, } \\
\text { total } \\
(\%)\end{array}$ \\
\hline FC-318 & 0.62 & 45 & 6.0 & 48 & 100 & 660 & 10 & 0.04 & 1.9 & 22 & 27 & 30 & 0.31 \\
\hline FC-324 & 1.0 & 28 & $<5.0$ & 34 & 74 & 2,600 & 7.3 & .02 & 1.2 & 14 & 20 & 35 & .23 \\
\hline FC-334 & .44 & 50 & $<5.0$ & 39 & 4.4 & 37 & 8.2 & .03 & 2.2 & 27 & 26 & 41 & .12 \\
\hline FC-343 & .17 & 66 & $<5.0$ & 44 & 21 & 23 & 16 & .05 & 2.2 & 34 & 44 & 28 & .16 \\
\hline FC-355 & .98 & 36 & $<5.0$ & 32 & 22 & 62 & 8.3 & .03 & 1.3 & 17 & 25 & 100 & .36 \\
\hline FC-697 & .23 & 28 & $<5.0$ & 27 & 52 & 3,600 & 4.5 & $<.02$ & 1.0 & 12 & 14 & 22 & .16 \\
\hline FC-819 & .23 & 68 & $<5.0$ & 56 & 18 & 630 & 15 & .05 & 3.0 & 34 & 32 & 40 & .52 \\
\hline FC-836 & .18 & 72 & $<5.0$ & 31 & 11 & 1,000 & 11 & .03 & 2.0 & 33 & 30 & 29 & .34 \\
\hline FC- 853 & 1.9 & 50 & $<5.0$ & 22 & 270 & 180 & 7.7 & .02 & 2.8 & 22 & 16 & 44 & .13 \\
\hline
\end{tabular}


Table 6. Chemical analysis of total extractible from samples from uranium waste dumps for selected major-ion and trace-element concentrations, Red, White, and Fry Canyons, southeastern Utah, 2007.-Continued

[Samples were analyzed at the U.S. Geological Survey Geologic Discipline Laboratory, Denver, Colorado. Site ID, site identification; MDT, Mountain Daylight Time; DD.dddd, Degrees.decimal degrees; \%, percent; ppm, parts per million; FC, Fry Canyon area; <, less than lower reporting limit]

\begin{tabular}{|c|c|c|c|c|c|c|c|c|c|c|c|c|}
\hline Site ID & $\begin{array}{c}\text { Manganese, } \\
\text { total } \\
\text { (ppm) }\end{array}$ & $\begin{array}{c}\text { Mercury, } \\
\text { total } \\
\text { (ppm) }\end{array}$ & $\begin{array}{l}\text { Molybdenum, } \\
\text { total } \\
\text { (ppm) }\end{array}$ & $\begin{array}{c}\text { Nickel, } \\
\text { total } \\
\text { (ppm) }\end{array}$ & $\begin{array}{c}\text { Niobium, } \\
\text { total } \\
\text { (ppm) }\end{array}$ & $\begin{array}{c}\text { Phosphorus, } \\
\text { total } \\
\text { (ppm) }\end{array}$ & $\begin{array}{c}\text { Potassium, } \\
\text { total } \\
(\%)\end{array}$ & $\begin{array}{c}\text { Rubidium, } \\
\text { total } \\
\text { (ppm) }\end{array}$ & $\begin{array}{l}\text { Scandium, } \\
\text { total } \\
\text { (ppm) }\end{array}$ & $\begin{array}{c}\text { Selenium, } \\
\text { total } \\
\text { (ppm) }\end{array}$ & $\begin{array}{c}\text { Silver, } \\
\text { total } \\
\text { (ppm) }\end{array}$ & $\begin{array}{c}\text { Sodium, } \\
\text { total } \\
(\%)\end{array}$ \\
\hline FC-318 & 210 & 0.01 & 12 & 61 & 7.4 & 420 & 1.2 & 56 & 9.7 & 0.50 & $<1.0$ & 0.08 \\
\hline FC-334 & 26 & .01 & 30 & 5.5 & 5.1 & 290 & 0.69 & 30 & 6.4 & .30 & $<1.0$ & .07 \\
\hline FC-343 & 110 & .02 & 13 & 16 & 11 & 370 & 1.2 & 71 & 11 & .50 & $<1.0$ & .11 \\
\hline FC-355 & 200 & .01 & 21 & 17 & 5.2 & 280 & 1.1 & 40 & 5.5 & .90 & $<1.0$ & .04 \\
\hline FC-721 & 270 & .08 & 16 & 33 & 8.5 & 690 & 2.6 & 110 & 12 & .40 & $<1.0$ & .60 \\
\hline FC-765 & 220 & $<.01$ & 1.2 & 23 & 3.3 & 190 & 0.62 & 27 & 4.5 & .20 & $<1.0$ & .05 \\
\hline FC-771 & 340 & .02 & 3.9 & 46 & 8.1 & 490 & 1.7 & 77 & 13 & .30 & $<1.0$ & .36 \\
\hline FC-772 & 130 & .01 & 16 & 27 & 4.8 & 250 & 0.91 & 33 & 6.0 & 2.5 & 3.0 & .25 \\
\hline FC-775 & 150 & .01 & 8.9 & 22 & 8.5 & 400 & 1.5 & 59 & 11 & .50 & $<1.0$ & .31 \\
\hline FC-853 & 240 & .03 & 8.7 & 100 & 3.7 & 410 & 0.77 & 29 & 5.9 & .30 & $<1.0$ & .09 \\
\hline
\end{tabular}

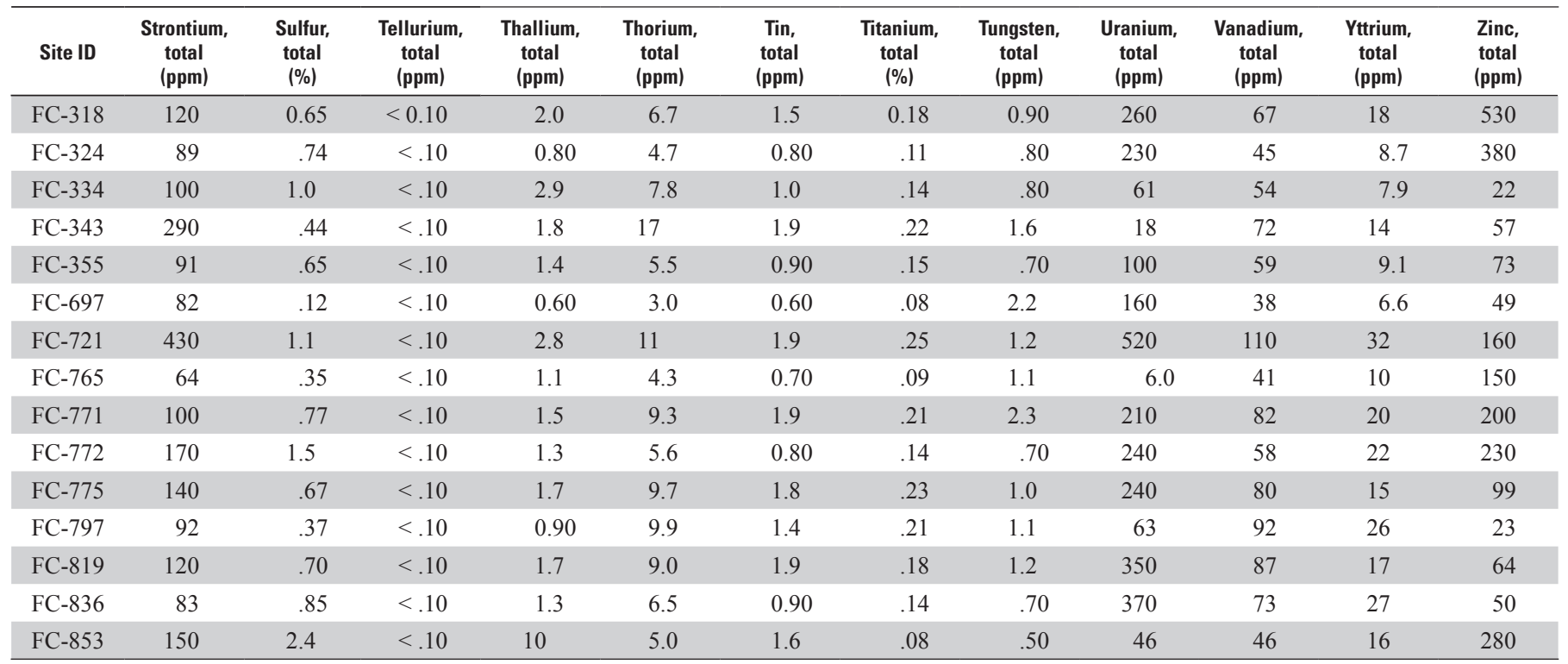




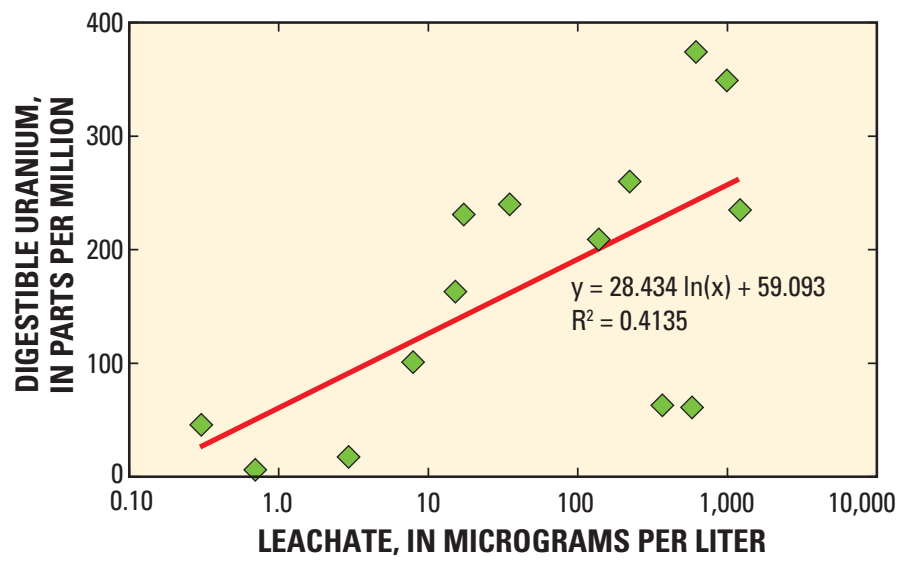

Figure 20. Relation between leachate and total digestible concentrations for uranium waste dump samples, Red, White, and Fry Canyons, southeastern Utah, 2007.

Table 7. Total extractible uranium concentration calculated from leachate concentration from uranium waste dump samples, Red, White, and Fry Canyons, southeastern Utah, 2007.

[Samples were analyzed at the U.S. Geological Survey Geologic Discipline Laboratory, Denver, Colorado. Site ID, site identification; MDT, Mountain Daylight Time; DD.dddd, Degrees.decimal degrees; ug/L, micrograms per liter; ppm, parts per million; FC, Fry Canyon area]

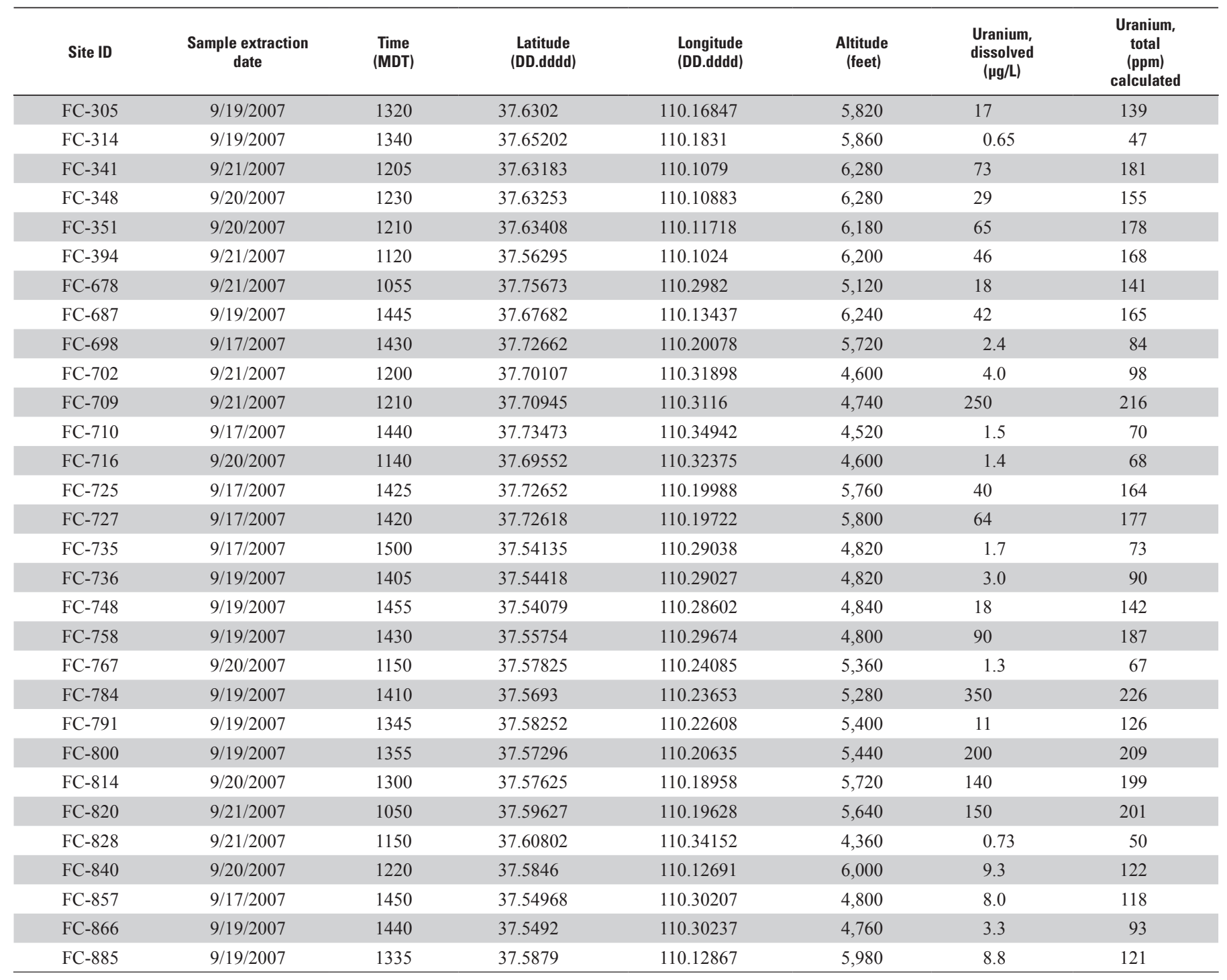


area background samples. The waste rock sites do not support significant plant life and have an average leachate uranium concentration of $131 \mu \mathrm{g} / \mathrm{L}$, while adjacent areas to the piles with significant plant life and potential for livestock forage have an average leachate uranium concentration of $7.6 \mu \mathrm{g} / \mathrm{L}$. Because the natural background for the area is approximately 5 percent of the average uranium concentration found in the waste rock piles, radionuclide propagation by local plant life uptake was not considered.

Concentrations of uranium were converted to activity of uranium-238, as this is the most abundant naturally occurring isotope: 99.27 percent of natural uranium is uranium-238. Natural uranium consists of uranium-234, -235, and -238 in ratios corresponding to natural isotopic abundances which are less than 0.01 percent, 0.72 percent, and 99.27 percent, respectively. The uranium decay series considered includes the decay chain of uranium-238 to thorium-230. Radium-226 and its associated short-lived decay chain were considered as a separate radionuclide series. Given the three exposure pathways considered, radium-226 and its associated decay chain poses the greatest risk to human health shown by the model's sensitivity to radium-226 concentrations.

Due to the particular use in the area, some of the assumptions about the parameters used by RESRAD have been altered to fit the site conditions. The primary use of the area appears to be recreational, off-road activity by riders of all-terrain vehicles (ATV). Two primary use scenarios were considered in determining the amount of annual exposure a visitor experiences. The first scenario considered the duration of activities of an individual that lives near the area and visits the site periodically during any given year. The total annual visitation by an individual to the site was 4.6 days per year based on calculations made for daily duration of outdoor activities by a young adult (U.S. Environmental Protection Agency, 1997b, table 15.3). The second scenario considered the duration of time spent on the site by an individual who is using the area as a recreation destination and spends 7 to 14 days on the site, the latter equal to the maximum annual recreational occupancy of a site by the BLM. In all scenarios, RESRAD was setup to inspect three exposure pathways appropriate to the usage scenarios that were identified: (a) external gamma exposure, (b) inhalation exposure, and (c) soil ingestion exposure.

Modifications to default data used by RESRAD to calculate an annual radiation dosage were made in the "Occupancy" and "Ingestion: Dietary" subsections of modifiable data; in all other subsections, default data was used (Yu and others, 1993). In the "Occupancy" subsection, an inhalation rate of $14,016 \mathrm{~m}^{3} /$ year was used instead of the default of $8,400 \mathrm{~m}^{3} /$ year to reflect moderate activities by an individual either riding an ATV or involved in outdoor activities (U.S. Environmental Protection Agency, 1997b, table 5.23). The mass loading for inhalation was set to $0.0004 \mathrm{~g} / \mathrm{m}^{3}$ over the default $0.0001 \mathrm{~g} /$ $\mathrm{m}^{3}$ to reflect the increased dust present on unpaved roads while ATV's are in use (Yu and others, 1993, chap. 35). The indoor time fraction was set to 0.0 instead of the default of 0.5 , inplying that the individual spends their entire time on the site outdoors. The outdoor time fraction was set appropriate to the annual exposure duration that was being considered: 0.013 for 4.6 days per year, 0.019 for 7.0 days per year, and 0.038 for 14.0 days per year. Outdoor time fractions are expressed as fractions of a one year period. In the "Ingestion: Dietary" subsection, a soil ingestion rate of $73 \mathrm{~g} /$ year was used instead of the default of $36.5 \mathrm{~g} /$ year to represent an average rate for all human age groups (U.S. Environmental Protection Agency, 1997b, table 4.23).

The resulting soil screening levels for combined uranium and radium for a maximum radiation dosage of $15 \mathrm{mrem} / \mathrm{year}$ are $96 \mathrm{pCi} / \mathrm{g}$ radium for 4.6 days per year, $66 \mathrm{pCi} / \mathrm{g}$ radium for 7.0 days per year, and $33 \mathrm{pCi} / \mathrm{g}$ radium for 14.0 days per year. At radium-uranium secular equilibrium, three sites, FC-721, FC-819, and FC-836, exceeded the 4.6 days-peryear SSL for radium. Ten additional sites, FC-318, FC-324, FC-709, FC-771, FC-772, FC-775, FC-784, FC-800, FC-814, and FC-820, exceeded the 7.0 days-per-year SSL for a total of thirteen sites. Eighteen additional sites, FC-305, FC-341, FC-348, FC-351, FC-355, FC-394, FC-678, FC-687, FC-697, FC-702, FC-725, FC-727, FC-748, FC-758, FC-791, FC-840, FC-857, and FC-885, exceeded the 14.0 days-per-year SSL for a total of 31 sites. At a radium-uranium activity ratio of 0.57 , one site, FC-721, exceeded the 4.6 days-per-year SSL for radium. Two additional sites, FC-819 and FC-836, exceeded the 7.0 days-per-year SSL for a total of three sites. Fourteen additional sites, FC-318, FC-324, FC-341, FC-351, FC-709, FC-727, FC-758, FC-771, FC-772, FC-775, FC-784, FC-800, FC-814, and FC-820, exceeded the 14.0 days-per-year SSL for a total of fifteen sites (table 8).

Two assumptions were made when calculating the SSL values using RESRAD. One was that radium and uranium were at secular equilibrium, the other that the radium-uranium activity ratio was 0.57 . When secular equilibrium was assumed, 31 sites exceeded a SSL and the majority, 16, were located in Red Canyon. At a radium-uranium activity ratio of $0.57,17$ sites exceeded a SSL and the majority, 12, were also located in Red Canyon. None of the sites located on Map 5 exceeded the calculated SSLs (fig. 6).

\section{Future Work}

Many uranium waste dumps in Red, White, and Fry Canyons contain elevated concentrations of trace elements as indicated from results of leachate analyses. On the basis of factors that include elevated leachate concentrations, basin drainage area, slope, and soil screening level exceedance, the following sites should be investigated further to assess potential risks to human health and the surrounding ecosystem: FC-318, FC-341, FC-351, FC-355, FC-394, FC-702, FC-709, FC-721, FC-727, FC-758, FC-771, FC-772, FC-775, FC-784, FC-800, FC-814, FC-819, FC-820, and FC-836. 
Table 8. Radium concentrations calculated from total extractible uranium concentrations from uranium waste dump samples, Red, White and Fry Canyons, southeastern Utah, 2007.

[Samples were analyzed at the U.S. Geological Survey Geologic Discipline Laboratory, Denver, Colorado. Site ID, site identification; MDT, Mountain Daylight Time; DD.dddd, Degrees.decimal degrees; ppm, parts per million; \%, percent; pCi/g, picocuries per gram; FC, Fry Canyon area]

\begin{tabular}{|c|c|c|c|c|c|c|c|c|c|}
\hline Site ID & $\begin{array}{c}\begin{array}{c}\text { Sample } \\
\text { extraction } \\
\text { date }\end{array} \\
\end{array}$ & $\begin{array}{c}\text { Time } \\
\text { (MDT) }\end{array}$ & $\begin{array}{l}\text { Latitude } \\
\text { (DD.dddd) }\end{array}$ & $\begin{array}{l}\text { Longitude } \\
\text { (DD.dddd) }\end{array}$ & $\begin{array}{c}\text { Altitude } \\
\text { (feet) }\end{array}$ & $\begin{array}{l}\text { Uranium, total } \\
(\mathrm{ppm})\end{array}$ & $\begin{array}{l}\text { Uranium, as } \\
\text { U-238 (pCi/g) }\end{array}$ & $\begin{array}{c}\text { Radium, at } \\
100 \% \text { of } U \\
(\mathrm{pCi} / \mathrm{g})\end{array}$ & $\begin{array}{c}\text { Radium, at } 57 \% \\
\text { of } \mathrm{U} \\
(\mathrm{pCi} / \mathrm{g})\end{array}$ \\
\hline FC-318 & $9 / 21 / 2007$ & 1045 & 37.66235 & 110.1984 & 5,660 & 260 & 87.5 & 87.5 & 49.9 \\
\hline FC-324 & $9 / 21 / 2007$ & 1140 & 37.63702 & 110.11743 & 6,140 & 230 & 77.4 & 77.4 & 44.1 \\
\hline FC-334 & $9 / 20 / 2007$ & 1240 & 37.6311 & 110.11207 & 6,220 & 61 & 20.5 & 20.5 & 11.7 \\
\hline $\mathrm{FC}-343$ & $9 / 20 / 2007$ & 1155 & 37.62942 & 110.10252 & 6,360 & 18 & 6.1 & 6.1 & 3.5 \\
\hline $\mathrm{FC}-355$ & $9 / 20 / 2007$ & 1225 & 37.6337 & 110.1177 & 6,100 & 100 & 33.7 & 33.7 & 19.2 \\
\hline FC-697 & $9 / 17 / 2007$ & 1445 & 37.67545 & 110.22558 & 5,280 & 160 & 53.9 & 53.9 & 30.7 \\
\hline FC-721 & 9/19/2007 & 1435 & 37.55278 & 110.28272 & 4,880 & 520 & 175.1 & 175.1 & 99.8 \\
\hline $\mathrm{FC}-765$ & $9 / 21 / 2007$ & 1105 & 37.60642 & 110.33933 & 4,400 & 6 & 2.0 & 2.0 & 1.2 \\
\hline FC-771 & $9 / 19 / 2007$ & 1415 & 37.5788 & 110.23853 & 5,280 & 210 & 70.7 & 70.7 & 40.3 \\
\hline $\mathrm{FC}-772$ & $9 / 20 / 2007$ & 1245 & 37.57905 & 110.2387 & 5,280 & 240 & 80.8 & 80.8 & 46.1 \\
\hline FC-775 & $9 / 20 / 2007$ & 1145 & 37.57822 & 110.24068 & 5,360 & 240 & 80.8 & 80.8 & 46.1 \\
\hline FC-797 & $9 / 21 / 2007$ & 1155 & 37.5814 & 110.20973 & 5,460 & 63 & 21.2 & 21.2 & 12.1 \\
\hline FC-819 & $9 / 19 / 2007$ & 1450 & 37.5975 & 110.19318 & 5,680 & 350 & 117.8 & 117.8 & 67.2 \\
\hline FC-836 & $9 / 21 / 2007$ & 1110 & 37.58912 & 110.15163 & 5,920 & 370 & 124.6 & 124.6 & 71.0 \\
\hline FC-853 & $9 / 21 / 2007$ & 1040 & 37.76457 & 110.36922 & 4,280 & 46 & 15.5 & 15.5 & 8.8 \\
\hline \multicolumn{10}{|c|}{ Uranium concentrations below were calculated from regression equation } \\
\hline FC-305 & $9 / 19 / 2007$ & 1320 & 37.6302 & 110.16847 & 5,820 & 139 & 46.8 & 46.8 & 26.7 \\
\hline FC-314 & $9 / 19 / 2007$ & 1340 & 37.65202 & 110.1831 & 5,860 & 47 & 15.8 & 15.8 & 9.0 \\
\hline FC-341 & $9 / 21 / 2007$ & 1205 & 37.63183 & 110.1079 & 6,280 & 181 & 60.9 & 60.9 & 34.7 \\
\hline FC-348 & 9/20/2007 & 1230 & 37.63253 & 110.10883 & 6,280 & 155 & 52.2 & 52.2 & 29.7 \\
\hline FC-351 & $9 / 20 / 2007$ & 1210 & 37.63408 & 110.11718 & 6,180 & 178 & 59.9 & 59.9 & 34.2 \\
\hline FC-394 & $9 / 21 / 2007$ & 1120 & 37.56295 & 110.1024 & 6,200 & 168 & 56.6 & 56.6 & 32.2 \\
\hline FC-678 & $9 / 21 / 2007$ & 1055 & 37.75673 & 110.2982 & 5,120 & 141 & 47.5 & 47.5 & 27.1 \\
\hline FC-687 & 9/19/2007 & 1445 & 37.67682 & 110.13437 & 6,240 & 165 & 55.6 & 55.6 & 31.7 \\
\hline FC-698 & $9 / 17 / 2007$ & 1430 & 37.72662 & 110.20078 & 5,720 & 84 & 28.3 & 28.3 & 16.1 \\
\hline FC-702 & $9 / 21 / 2007$ & 1200 & 37.70107 & 110.31898 & 4,600 & 98 & 33.0 & 33.0 & 18.8 \\
\hline FC-709 & $9 / 21 / 2007$ & 1210 & 37.70945 & 110.3116 & 4,740 & 216 & 72.7 & 72.7 & 41.5 \\
\hline FC-710 & $9 / 17 / 2007$ & 1440 & 37.73473 & 110.34942 & 4,520 & 70 & 23.6 & 23.6 & 13.4 \\
\hline FC-716 & $9 / 20 / 2007$ & 1140 & 37.69552 & 110.32375 & 4,600 & 68 & 22.9 & 22.9 & 13.1 \\
\hline FC-725 & $9 / 17 / 2007$ & 1425 & 37.72652 & 110.19988 & 5,760 & 164 & 55.2 & 55.2 & 31.5 \\
\hline $\mathrm{FC}-727$ & $9 / 17 / 2007$ & 1420 & 37.72618 & 110.19722 & 5,800 & 177 & 59.6 & 59.6 & 34.0 \\
\hline FC-735 & $9 / 17 / 2007$ & 1500 & 37.54135 & 110.29038 & 4,820 & 73 & 24.6 & 24.6 & 14.0 \\
\hline FC-736 & $9 / 19 / 2007$ & 1405 & 37.54418 & 110.29027 & 4,820 & 90 & 30.3 & 30.3 & 17.3 \\
\hline FC-748 & $9 / 19 / 2007$ & 1455 & 37.54079 & 110.28602 & 4,840 & 142 & 47.8 & 47.8 & 27.3 \\
\hline $\mathrm{FC}-758$ & $9 / 19 / 2007$ & 1430 & 37.55754 & 110.29674 & 4,800 & 187 & 63.0 & 63.0 & 35.9 \\
\hline FC-767 & $9 / 20 / 2007$ & 1150 & 37.57825 & 110.24085 & 5,360 & 67 & 22.6 & 22.6 & 12.9 \\
\hline FC-784 & $9 / 19 / 2007$ & 1410 & 37.5693 & 110.23653 & 5,280 & 226 & 76.1 & 76.1 & 43.4 \\
\hline FC-791 & $9 / 19 / 2007$ & 1345 & 37.58252 & 110.22608 & 5,400 & 126 & 42.4 & 42.4 & 24.2 \\
\hline FC-800 & $9 / 19 / 2007$ & 1355 & 37.57296 & 110.20635 & 5,440 & 209 & 70.4 & 70.4 & 40.1 \\
\hline FC-814 & $9 / 20 / 2007$ & 1300 & 37.57625 & 110.18958 & 5,720 & 199 & 67.0 & 67.0 & 38.2 \\
\hline FC-820 & $9 / 21 / 2007$ & 1050 & 37.59627 & 110.19628 & 5,640 & 201 & 67.7 & 67.7 & 38.6 \\
\hline FC-828 & $9 / 21 / 2007$ & 1150 & 37.60802 & 110.34152 & 4,360 & 50 & 16.8 & 16.8 & 9.6 \\
\hline FC-840 & $9 / 20 / 2007$ & 1220 & 37.5846 & 110.12691 & 6,000 & 122 & 41.1 & 41.1 & 23.4 \\
\hline FC-857 & $9 / 17 / 2007$ & 1450 & 37.54968 & 110.30207 & 4,800 & 118 & 39.7 & 39.7 & 22.6 \\
\hline FC-866 & 9/19/2007 & 1440 & 37.5492 & 110.30237 & 4,760 & 93 & 31.3 & 31.3 & 17.8 \\
\hline FC-885 & 9/19/2007 & 1335 & 37.5879 & 110.12867 & 5,980 & 121 & 40.7 & 40.7 & 23.2 \\
\hline
\end{tabular}




\section{References Cited}

Briggs, P.H., 2002, The determination of forty elements in geological and botanical samples by inductively coupled plasma-atomic emission spectrometry, in Taggart, J.E., Jr., ed., Analytical methods for chemical analysis of geologic and other materials: U.S. Geological Survey Open-File Report 02-223-G, p. G1-G18.

Brown, Z.A., O'Leary, R.M., Hageman, P.L., and Crock, J.G., 2002, Mercury in water, geologic, and plant materials by continuous flow-cold vapor-atomic absorption spectrometry, in Taggart, J.E., Jr., ed., Analytical methods for chemical analysis of geologic and other materials: U.S. Geological Survey Open-File Report 02-223-M, p. M1-M9.

Crock, J.G., Lichte, F.E., and Briggs, P.H., 1983, Determination of elements in National Bureau of Standards geological reference materials SRM 278 obsidian and SRM 688 basalt by inductively coupled plasma-atomic emission spectroscopy: Geostandards Newsletter, v. 7, no. 2, p. 335-340.

Hageman, P.L., and Briggs, P.H., 2000, A simple field leach test for rapid screening and qualitative characterization of mine uranium waste dump material on abandoned mine lands: Proceedings from the Fifth International Conference on Acid Rock Drainage, Denver, Colorado, May 21-24, 2000, p. 1463-1475.

Hageman, P.L., Brown, Z.A., and Welsch, E., 2002, Arsenic and selenium by flow injection or continuous flow-hydride generation-atomic absorption spectrophotometry, in Taggart, J.E., Jr., ed., Analytical methods for chemical analysis of geologic and other materials: U.S. Geological Survey OpenFile Report 02-223-L, p. L1-L7.

Hintze, L.F., Willis, G.C., Laes, D.Y.M., Sprinkle, D.A., and Brown, K.D., 1996, Digital compilation of geologic map of Utah: U.S. Geological Survey Digital Data Series 41.

Kenney, T.A., Wilkowske, C.D., and Wright, S.J., 2007, Methods for estimating magnitude and frequency of peak flows for natural streams in Utah: U.S. Geological Survey Scientific Investigations Report 2007-5158, 28 p.

Natural Resources Conservation Service, 1998, Utah Annual Precipitation [map], scale: 1:1,000,000, Source 1008501.
Ries, K.G., III, Steeves, P.A., Coles, J.D., Rea, A.H., and Stewart, D.W., 2004, StreamStats: A U.S. Geological Survey web application for stream information: U.S. Geological Survey Fact Sheet 2004-3115.

Smith, K.S., Ramsey, C.A., and Hageman, P.L., 2000, Sampling strategy for the rapid screening of mine-waste dumps on abandoned mine lands: Proceedings from the Fifth International Conference on Acid Rock Drainage, Denver, Colorado, May 21-24, 2000, p. 1453-1461.

Stearn, T.W., and Stieff, L.R., 1959, Geochemistry and mineralogy of the Colorado Plateau uranium ores: Part 13. Radium-uranium equilibrium and radium-uranium ages of some secondary minerals, U.S. Geological Survey Professional Paper 320, 1959. p. 151-156.

U.S. Environmental Protection Agency, 1997a, Memorandum -Establishment of cleanup levels for CERCLA sites with radioactive contamination. OSWER No. 9200.4-18. Washington, DC, August 1997.

U.S. Environmental Protection Agency, 1997b, Exposure factors handbook. Washington, DC, EPA/600/P-95/002F a-c, 1997.

U.S. Environmental Protection Agency, 2007a, Current national recommended water quality criteria for aquatic life: U.S. Environmental Protection Agency database, accessed January 21, 2007, at http://www.epa.gov/waterscience/ criteria/wqcriteria.html.

U.S. Environmental Protection Agency, 2007b, Drinking water contaminants: U.S. Environmental Protection Agency database, accessed January 22, 2007, at http://www.epa.gov/ safewater/contaminants/index.html.

Wilde, F.D., ed., 2004, Cleaning of equipment for water sampling (ver. 2.0): U.S. Geological Survey Techniques of Water-Resources Investigations, book 9, chap. A3, accessed October 10, 2006, at http://pubs.water.usgs.gov/twri9A3/.

Yu, C., Loureiro, C., Cheng, J.J., Jones, L.G., Wang, Y.Y., Chia, Y.P., and Faillance, E., 1993, Data collection handbook to support modeling impacts of radioactive material in soil. Environmental Assessment and Information Sciences Division, Argonne National Laboratory, Argonne, Illinois, 1993. 



\section{Appendix A. Chemical Analysis of Leachate Samples from Uranium Waste Dump Sites, Background Sites, and Streambed Sediment Sites, for Major-Ion and Trace-Element Concentrations, Red, White, and Fry Canyons, Southeastern Utah, 2007.}

Appendix A is a Microsoft Excel spreadsheet, a separate, associated document from this report. This table is titled, Chemical analysis of leachate samples from uranium waste dump sites, background sites, and streambed sediment sites, for major-ion and trace-element concentrations, Red, White, and Fry Canyons, southeastern Utah, 2007. This table is available online and is recommended to be downloaded with the main report, Scientific Investigations Report 2010-5108. Place this Excel document on the same file level as the main report. This will enable any links associated with the document to function between the main report and the Excel file. 




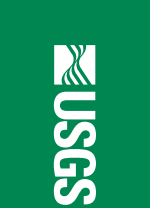

II 